\title{
From Sommerfeld Diffraction Problems to Operator Factorisation
}

\author{
Dedicated to Professor Erhard Meister on the occasion of his $90^{\text {th }}$ anniversary
}

FRANK-OLME SPECK*

\begin{abstract}
This article presents a brief survey devoted to the interplay between diffraction and operator theory, particularly between the so-called canonical diffraction problems (exemplified by Sommerfeld half-plane problems) on one hand and operator factorisation theory on the other hand. It is shown how operator factorisation concepts appear in a natural way from applications and how they can help to find solutions rigorously in case of well-posed problems or how to normalise problems by an adequate change of function spaces.
\end{abstract}

Keywords: Sommerfeld problem, diffraction, Wiener-Hopf method, Wiener-Hopf operator, factorisation, intermediate space, generalised inverse, operator relation.

2010 Mathematics Subject Classification: 47A68, 47B35.

\section{INTRODUCTION}

The original Sommerfeld diffraction problem has been solved in closed analytical form firstly by Arnold Sommerfeld in 1896 with the help of Fresnel integrals [84, 103]. An analytical solution with the so-called Wiener-Hopf technique was found in the 1940s, see [58, 86, 121]. It allowed a better understanding of the asymptotic behaviour of the solution, see also [66].

Another fourty years later, it has been proved to be well posed in the sense of Hadamard $[47,68]$ in certain Sobolev space settings [77, 107]. Moreover, the resolvent operator has been identified as an explicit formula based upon operator factorisation in the sense of Shinbrot in the context of a wider class of problems [33,101,106,107]. We describe the corresponding basic ideas in the next two sections. It leads us to a detailed study of the Wiener-Hopf factorisation of matrix functions which will be discussed in Section 4. In Section 5, a general concept of operator factorisation in linear boundary value and transmission problems is briefly outlined. Section 6 is devoted to the choice of adequate function(al) spaces and symbol classes occurring from diffraction theory. In Section 7, we expose some logical sharp concepts of equivalence and reduction coming up with operator factorisation and with the aim to construct resolvent operators, i.e., inverses (or generalised inverses) of operators associated with linear boundary value and transmission problems. Section 8 presents a glance at concepts which are more general than operator factorisation, namely given by certain operator relations with applications in diffraction theory. In the last section, we demonstrate the usefulness of the preceding concepts once again by the generalisation of Sommerfeld problems from two to higher dimensions. At

Received: 16 September 2019; Accepted: 11 November 2019; Published Online: 12 November 2019

*Corresponding author: Frank-Olme Speck; fspeck@tecnico.pt

DOI: $10.33205 / \mathrm{cma} .620578$ 
the end of each section, the reader may find a short historical review of selected relevant publications.

The operator theoretical approach has the advantage of a compact presentation of results simultaneously for wide classes of diffraction problems which are analytically solvable by the Wiener-Hopf method and it gives a different and deeper understanding of the solution procedures. An interpretation in the physical sense, such as a consideration of the solution of a boundary value problem as the perturbed wave field in the diffraction of time-harmonic wave propagation, can be found in the cited literature, see $[69,70,71,72]$, for instance.

The main objective of this article is to demonstrate how real applications naturally guide us to operator factorisation concepts and how useful those are to simplify and to strengthen the reasoning in the applications. The exposition of these concepts will be accompanied by concrete problems closely connected with the Rawlins problem $[92,93]$.

\section{FROM THE CLASSICAL WIENER-HOPF TECHNIQUE TO OPERATOR FACTORISATION}

First, we consider the linear (non-singular) convolution equation on the real half-line

$$
\mathcal{W} f(x)=a f(x)-\int_{0}^{\infty} K(x-y) f(y) d y=g(x) \quad, \quad x>0 .
$$

Herein $a \in \mathbb{C} \backslash\{0\}, K \in L^{1}(\mathbb{R})$ and $g \in L^{p}\left(\mathbb{R}_{+}\right), p \in[1, \infty]$ are (arbitrarily) given and $f \in L^{p}\left(\mathbb{R}_{+}\right)$ is unknown, $\mathbb{R}_{+}=(0, \infty)$. It is well known that $\mathcal{W}$ defines a bounded linear operator acting in a Banach space $X=L^{p}\left(\mathbb{R}_{+}\right)$

$$
\mathcal{W}: L^{p}\left(\mathbb{R}_{+}\right) \rightarrow L^{p}\left(\mathbb{R}_{+}\right),
$$

in brief $\mathcal{W} \in \mathcal{L}\left(L^{p}\left(\mathbb{R}_{+}\right)\right)$, known as classical Wiener-Hopf operator (WHO) [42, 62, 79]. For simplicity, we abbreviate $L^{p}=L^{p}(\mathbb{R})$ and choose $a=1$ and $p=2$ in this discussion, focusing the scalar case (instead of systems of equations [43]).

Now, let us look at the steps of the Wiener-Hopf technique $[70,86]$ from the viewpoint of operator theory. Certainly one likes to find out whether the equation (2.1) is uniquely solvable and, in this case, the solution $f$ depends continuously on the given function $g$, (i.e., whether (2.1) is well-posed) and also to obtain an explicit analytical formula for the solution, in brief to determine $W^{-1} \in \mathcal{L}\left(L^{2}\left(\mathbb{R}_{+}\right)\right)$if possible. To this end, we need some notation. We denote in (2.1) the convolution operator on the full real line by $A$ and obtain with the help of the Fourier transformation $\mathcal{F}$ from the convolution theorem that

$$
\begin{aligned}
A & =I+K *=\mathcal{F}^{-1} \Phi \cdot \mathcal{F} \quad: \quad L^{2} \rightarrow L^{2}, \\
\Phi & =1+\mathcal{F} K \in L^{\infty}(\mathbb{R}), \\
\mathcal{F} K(\xi) & =\frac{1}{\sqrt{2 \pi}} \int_{\mathbb{R}} e^{i x \xi} K(x) d x \quad, \quad \xi \in \mathcal{R} .
\end{aligned}
$$

Hence, the classical Wiener-Hopf operator can be written as

$$
\mathcal{W}=r_{+} A \ell_{0}=L^{2}\left(\mathbb{R}_{+}\right) \rightarrow L^{2}\left(\mathbb{R}_{+}\right)
$$

where $r_{+}$and $\ell_{0}$ denote the restriction and zero extension operator, respectively:

$$
r_{+}: L^{2}(\mathbb{R}) \rightarrow L^{2}\left(\mathbb{R}_{+}\right) \quad, \quad \ell_{0}: L^{2}\left(\mathbb{R}_{+}\right) \rightarrow L^{2}(\mathbb{R}),
$$

which are bounded linear operators. Obviously, we have

$$
r_{+} \ell_{0}=I_{L^{2}\left(\mathbb{R}_{+}\right)}, \quad P=\ell_{0} r_{+} \in \mathcal{L}\left(L^{2}\right),
$$


where $P$ is a projector acting in $L^{2}$, i.e., $P \in \mathcal{L}\left(L^{2}\right), P^{2}=P$, with image (or range) and kernel (or null space) given by

$$
\begin{aligned}
& \operatorname{im} P=L_{+}^{2}=\left\{f \in L^{2}: \operatorname{supp} f \subset \overline{\mathbb{R}_{+}}\right\}, \\
& \operatorname{ker} P=L_{-}^{2}=\left\{f \in L^{2}: \operatorname{supp} f \subset \overline{\mathbb{R}_{-}}\right\} .
\end{aligned}
$$

We shall say that $P$ projects onto im $P$ along ker $P$.

With the help of this basic notation the steps of the Wiener-Hopf technique can be described as follows. First, the equation (2.1) is extended by zero to an equation holding on the full line:

$$
A f_{+}=g_{+}+g_{-},
$$

where $g_{+}=\ell_{0} g \in L_{+}^{2}$ is known and $f_{+}=\ell_{0} g \in L_{+}^{2}, g_{-} \in L_{-}^{2}$ are unknown provided (2.1) is solvable.

In what follows the Fourier symbol $\Phi=1+\mathcal{F} K$ of $A$ plays a decisive role. Note that this function is also referred to as "Fourier transformed kernel" or simply as "kernel" in some papers such as [63] which has to be distinguished from the convolution kernel $K$ of the integral operator $A$ and from the kernel (or null space) of a linear operator like $A$ or $W$ (hence we avoid calling $\Phi$ a kernel).

Now, let us assume that $|\Phi|$ is bounded from below. Since the Fourier symbol $\Phi$ of $A$ belongs to the Wiener algebra $\mathbb{C} \oplus \mathcal{F} L^{1}$ (with $\Phi(\infty)=1$ ), it allows a factorisation [44, 62]

$$
\begin{aligned}
\Phi & =\Phi_{-} \zeta^{\kappa} \Phi_{+}, \\
\kappa & =\frac{1}{2 \pi} \int_{\mathbb{R}} d \arg \Phi(\xi) d \xi \in \mathbb{Z}, \quad \zeta(\xi)=\frac{\xi-i}{\xi+i}, \quad \xi \in \mathbb{R}, \\
\Phi_{ \pm} & =\exp \left\{\mathcal{F} \ell_{0} r_{ \pm} \mathcal{F}^{-1} \log \left(\zeta^{-\kappa} \Phi\right)\right\} .
\end{aligned}
$$

With the help of this factorisation one can continue considering the Fourier transformed equation of (2.8) (as common in the classical Wiener-Hopf technique [86]) or, alternatively, making directly use of the operator factorisation that results from (2.9):

$$
\begin{aligned}
A & =A_{-} C A_{+}, \\
A_{ \pm} & =\mathcal{F}^{-1} \Phi_{ \pm} \cdot \mathcal{F}, \quad C=\mathcal{F}^{-1} \zeta^{\kappa} \cdot \mathcal{F}: L^{2} \rightarrow L^{2} .
\end{aligned}
$$

All the three factors $A_{-}, C, A_{+}$are isomorphisms in $L^{2}$ (i.e., linear homeomorphisms, boundedly invertible operators), and the inverses are convolution operators with Fourier symbols $\Phi_{-}^{-1}, \zeta^{-\kappa}, \Phi_{+}^{-1}$ in the Wiener algebra and value 1 at infinity.

The next step in the Wiener-Hopf technique is a rearrangement of equation (2.8) which is different depending on whether $\kappa \geq 0$ or $\kappa \leq 0$ (and coinciding for $\kappa=0$ where $C=I$ ), namely

$$
\begin{aligned}
C A_{+} f_{+} & =A_{-}^{-1} g_{+}+A_{-}^{-1} g_{-} \quad, \quad \text { if } \kappa \geq 0, \\
A_{+} f_{+} & =C^{-1} A_{-}^{-1} g_{+}+C^{-1} A_{-}^{-1} g_{-}, \text {if } \kappa \leq 0 .
\end{aligned}
$$

Now, the classical Wiener-Hopf technique [86] works with holomorphy properties of the Fourier transformed terms of the equation and an additive decomposition of the first term on the right hand side which results in the same conclusion as applying the projector $P$ on the two 
equations, briefly

$$
\begin{aligned}
P C A_{+} f_{+} & =P A_{-}^{-1} g_{+}, \quad \text { if } \kappa \geq 0, \\
P A_{+} f_{+} & =P C^{-1} A_{-}^{-1} g_{+}, \quad \text { if } \kappa \leq 0 .
\end{aligned}
$$

This is just a consequence of the invariance properties of the factors: $A_{+}$maps im $P$ onto itself, $A_{-}$maps ker $P$ onto itself. Furthermore, in case of $\kappa \geq 0$, the middle factor $C$ maps im $P$ into itself, whilst $C^{-1}$ maps ker $P$ into itself, and in case of $\kappa \leq 0$, it's the other way around. These properties altogether are equivalent to the following formulas, so-called invariance properties of the factors:

$$
\begin{aligned}
P A_{+} P & =A_{+} P, \quad P A_{+}^{-1} P=A_{+}^{-1} P, \\
P A_{-} P & =P A_{-}, \quad P A_{-}^{-1} P=P A_{-}^{-1}, \\
P C P & =C P, \quad P C^{-1} P=P C^{-1}, \text { if } \kappa \geq 0, \\
P C P & =P C, \quad P C^{-1} P=C^{-1} P, \text { if } \kappa \leq 0 .
\end{aligned}
$$

If $\kappa \geq 0$, the equation (2.12) can be easily transformed with the help of the factor properties (2.13) into

$$
f_{+}=A_{+}^{-1} P C^{-1} P A_{-}^{-1} g_{+} \in L_{+}^{2} .
$$

In the original setting (2.1), we obtain

$$
f=r_{+} A_{+}^{-1} P C^{-1} P A_{-}^{-1} \ell_{0} g \in L^{2}\left(\mathbb{R}_{+}\right) .
$$

However, this formula holds only if the equation (2.1) is solvable (as we started from the assumption that (2.1) holds). Looking carefully at (2.11) one can find (for $\kappa>0$ ) a necessary solubility condition, namely $P A_{-}^{-1} g_{+} \in \operatorname{im} P C L_{+}^{2}$, which can be verified to be sufficient, as well.

Instead of this argumentation one can prove that (2.15) presents a left inverse of the operator $\mathcal{W}$ in (2.4) for all $\kappa \geq 0$ by the help of (2.13). Similarly one verifies that it gives a right inverse of $\mathcal{W}$ in the case of $\kappa \leq 0$.

Denoting these one-sided inverses by $\mathcal{W}^{-}$we have in the case $\kappa>0$ a (non-trivial) projector onto the image of $\mathcal{W}$ given by $\mathcal{W} \mathcal{W}^{-}$and in the case $\kappa<0$ a (non-trivial) projector along the kernel of $\mathcal{W}$ given by $\mathcal{W}^{-} \mathcal{W}$. So, we obtain as a common operator theoretical interpretation:

Theorem 2.1. Let $\mathcal{W}$ be given by (2.4) where $|\Phi|$ be bounded from below, i.e., $A \in \mathcal{L}\left(L^{2}(\mathbb{R})\right)$ is an isomorphism, and let $\mathcal{W}^{-}$be the operator from (2.15). Then, we have for

- $\kappa=0$ : Equation (2.1) is uniquely solvable by (2.15) (where $C=I$ ) for any $g \in L^{2}\left(\mathbb{R}_{+}\right)$.

- $\kappa>0$ : Equation (2.1) is solvable for a certain $g \in L^{2}\left(\mathbb{R}_{+}\right)$if and only if $g$ satisfies the condition

$$
\mathcal{W} \mathcal{W}^{-} g=r_{+} A_{-} P C P C^{-1} P A_{-}^{-1} \ell_{0} g=g .
$$

In this case, (2.15) presents the unique solution.

- $\kappa<0$ : Equation (2.1) is solvable for all $g \in L^{2}\left(\mathbb{R}_{+}\right)$. The general solution reads

$$
\begin{aligned}
f & =\mathcal{W}^{-} g+\left(I-\mathcal{W}^{-} \mathcal{W}\right) h, h \in L^{2}\left(\mathbb{R}_{+}\right) \\
\mathcal{W}^{-} \mathcal{W} & =r_{+} A_{+}^{-1} P C^{-1} P C P A_{+} \ell_{0} .
\end{aligned}
$$

Remark 2.1. Moreover one can conclude that $\mathcal{W}$ is a Fredholm operator, i.e., its defect numbers are finite: $\operatorname{dim} \operatorname{ker} W<\infty$, codim $\operatorname{im} W<\infty$. The solubility conditions in the case $\kappa>0$ can be written as $\kappa$ orthogonality conditions using a suitable basis of $L^{2}$. The kernel of $\mathcal{W}$ can also be explicitly written as $a-\kappa$-dimensional subspace of $L^{2}\left(\mathbb{R}_{+}\right)[42,62,79]$. Details are given later in (7.91) - (7.94). 
Remark 2.2. It is well known that the appearance of one-sided invertibility has a deeper background in the Theorem of Coburn, see [10, Section 2.6], which allows the conclusion that for certain classes of operators (such as classical scalar Toeplitz and Wiener-Hopf operators), the Fredholm property implies one-sided invertibility.

Hence the question arises if, in a general setting, a factorisation of $A$ with the factor properties (2.13) is not only sufficient for the one-sided invertibility of $\mathcal{W}$, but moreover if such a factorisation is necessary, as well. For clarity, let us first see the case where $\mathcal{W}$ is invertible and $C=I$ coming back to the general case later. To this end, we need the following notation.

Let $X$ be a Banach space, $A \in \mathcal{L}(X)$ and $P \in \mathcal{L}(X)$ a projector, and put $Q=I-P, P X=$ $\operatorname{im} P=\operatorname{ker} Q, Q X=\operatorname{im} Q=\operatorname{ker} P$ for convenience. Then

$$
W=\left.P A\right|_{P X} \quad: \quad P X \rightarrow P X
$$

is referred to as general Wiener-Hopf operator (WHO). If $A$ is an isomorphism, an operator pair $A_{+}, A_{-} \in \mathcal{L}(X)$ is said to be a strong (right) WH factorisation of $A$ with respect to $(X, P)$ if

$$
A=A_{-} A_{+}
$$

and the first two lines of the relations (2.13) are satisfied, i.e., $A_{+}$leaves $\operatorname{im} P$ invariant and $A_{-}$leaves ker $P$ invariant. As a standard situation in this general setting we shall work only with Banach spaces; other convenient frameworks could be topological vector spaces or Hilbert spaces.

Remark 2.3. The classical WHO (2.4) is not of the form (2.16) as $L^{2}\left(\mathbb{R}_{+}\right)$is not a subspace of $L^{2}(\mathbb{R})$. But $L^{2}\left(\mathbb{R}_{+}\right)$is isomorphic to the subspace $L_{+}^{2} \subset L^{2}(\mathbb{R})$, see (2.5) - (2.7). With the operator P of (2.6), we therefore have

$$
\begin{aligned}
& W=\left.\ell_{0} r_{+} A \ell_{0} r_{+}\right|_{L_{+}^{2}}=\ell_{0} \mathcal{W} r_{+}: L_{+}^{2} \rightarrow L_{+}^{2}, \\
& \mathcal{W}=\left.r_{+} P A\right|_{P X} \ell_{0}=r_{+} W \ell_{0}: L^{2}\left(\mathbb{R}_{+}\right) \rightarrow L^{2}\left(\mathbb{R}_{+}\right),
\end{aligned}
$$

i.e., the classical WHO is equivalent to an operator of the form of a general WHO (2.16).

Remember that two bounded linear operators $S, T$ acting in Banach spaces are said to be equivalent, if there are isomorphisms $E, F$ such that

$$
T=E S F,
$$

briefly written as

$$
T \sim S .
$$

Theorem 2.2 (of Devinatz and Shinbrot). Let the assumptions of (2.16) be fulfilled and $A$ be an isomorphism. Then, the WHO $W$ is boundedly invertible if and only if $A$ admits a strong WH factorisation with respect to $(X, P)$. In this case, the inverse of $W$ is given by

$$
W^{-1}=\left.A_{+}^{-1} P A_{-}^{-1}\right|_{P X} \quad: \quad P X \rightarrow P X .
$$

The proof is quite elementary, although not at all constructive. Sufficiency is verified with the help of the factor properties (2.13). Necessity is proved as follows: If $W$ is invertible in $\mathcal{L}(P X)$, then $P A+Q$ is invertible in $\mathcal{L}(X)$ because of the well-known relation

$$
P A P+Q=(I-P A Q)(P A+Q),
$$

where $P A P+Q$ is obviously invertible and $(I-P A Q)^{-1}=I+P A Q$. Now put just

$$
A=A_{-} A_{+}=A_{-}(P A+Q)
$$

and verify the factor properties, see [106] for details. 
This result was a cornerstone for the study of general Wiener-Hopf operators as given by (2.16) and moreover for general WHOs in an asymmetric space setting [106, 112] defined as follows.

Let $X, Y$ be Banach spaces, $A \in \mathcal{L}(X, Y)$ an isomorphism, $P_{1} \in \mathcal{L}(X), P_{2} \in \mathcal{L}(Y)$ two projectors, and put $Q_{j}=I-P_{j}, P_{1} X=\operatorname{im} P_{1}=\operatorname{ker} Q_{1}$ etc. for convenience. Then

$$
W \quad=\left.\quad P_{2} A\right|_{P_{1} X} \quad: \quad P_{1} X \rightarrow P_{2} Y
$$

is referred to as a general WHO (in asymmetric setting).

The case of one-sided invertible WHOs can be seen as a special case of generalised invertibility, i.e., for $T \in \mathcal{L}(X, Y)$ there exists an operator $T^{-}$such that

$$
T T^{-} T=T \text {. }
$$

This conception allows a unified discussion of many kinds of general WHOs and their realisations. It will be extended later in Section 7.

In what concerns Sommerfeld diffraction problems several modifications of the WHO (2.1) have to be considered, particularly:

- operators acting between different (Sobolev-like) functional spaces,

- matrix instead of scalar operators,

- more general Fourier symbol classes (rather than the Wiener algebra).

The need of these generalisations will be shown in the following Sections 3, 4, and 6, respectively.

\section{Historical remarks.}

Wiener-Hopf equations are named after Norbert Wiener and Eberhard Hopf who considered this kind of equations in 1931 (in German) [122]. Crucial progress in the solution of WH equations was presented in the fundamental paper by Mark Krein in 1958 (in Russian) [62] and for systems by Israel Gohberg and Mark Krein in the same year [43] (which was even published before the previous). Various function spaces were considered besides the Lebesgue spaces (with similar results) and also various symbol classes besides the Wiener algebra allowed similar results, because the Hilbert transformation is continuous in so-called decomposing algebras (including Hölder continuous functions). Other symbol classes (like the continuous functions) are not decomposing, the (formal) factors are not in the same class, see the books by I.C. Gohberg and I.A. Fel'dman [42] or Solomon Grigor'evich Mikhlin and Siegfried Prössdorf [79] and the most important article on generalised factorisation by I.B. Simonenko from 1968 [102].

The Wiener-Hopf technique (also called WH method or WH procedure) developed parallel to the previous advances in applications, mainly by British researchers, see the famous books of Ben Noble from 1958 [86], by Douglas Jones from 1964 [58], as well as the surveys on the classical WH method in applications by David Abrahams [2] and another one by Jane Lawrie and David Abrahams [63]. Further remarkable progress was obtained for instance in Canada by Albert Heins and Robert Allan Hurd (loc. cit.), in Russia, see the book of Lev Weinstein [121], and later in Turkey by Mithat Idemen [56], A. Hamit Serbest et al. [98].

General WHOs were introduced by Marvin Shinbrot in 1964 [101]. Theorem 2.5 appeared in a paper with Allan Devinatz in 1969 [33] firstly for separable Hilbert spaces with a rather complicated proof, a year after a more general result about one-sided invertibility of ring elements (instead of $P A P \in \mathcal{L}(X)$ ) that was already published by Grigorii Chebotarev in 1968 (in Russian) [29], under the name abstract WHO.

Some ideas about general WHOs appeared independently under the names truncation, projection, or compression of an operator, see the books [10, 44, 65], for instance. 
Also, in the context of pseudo-differential operators (acting in Sobolev spaces) we can find similar ideas and related results, see $[41,117]$. Some higher dimensional WH equations in applications have been addressed in $[45,75]$, for instance.

The connection between classical and general WHOs was firstly pointed out in [33, 75], see also $[71,72]$.

\section{THE WH EQUATIONS IN SOMMERFELD HALF-PLANE PROBLEMS}

A Sommerfeld half-plane problem is here referred to as to determine the solution $u$ of a boundary value problem for the Helmholtz equation

$$
\left(\Delta+k^{2}\right) u(x)=\frac{\partial^{2} u(x)}{\partial x_{1}^{2}}+\frac{\partial^{2} u(x)}{\partial x_{2}^{2}}+\frac{\partial^{2} u(x)}{\partial x_{3}^{2}}+k^{2} u(x)=0
$$

in the slit domain $\Omega=\mathbb{R}^{3} \backslash \Sigma$, where the screen $\Sigma$ is a half-plane $\Sigma=\left\{x=\left(x_{1}, x_{2}, x_{3}\right) \in\right.$ $\left.\mathbb{R}^{3}: x_{1} \geq 0, x_{2}=0\right\}$ on which the field $u$ is known, or its normal derivative or another linear boundary condition is prescribed on the two sides $\Sigma^{ \pm}$of the screen (corresponding with $x_{1} \geq$ $0, x_{2}= \pm 0$ ). The wave number $k$ is assumed to have a positive imaginary part throughout this paper. With the argument that in certain cases (as for a plane wave incoming perpendicularly to the edge of the screen) the solution will not depend on the third variable, the problem is modified to be a two-dimensional one, briefly written as

$$
\begin{aligned}
\left(\Delta+k^{2}\right) u & =0 \quad \text { in } \Omega, \\
T_{0, \Sigma^{ \pm}} u & =g \text { on } \Sigma^{ \pm}
\end{aligned}
$$

in case of the Sommerfeld-Dirichlet problem, with the trace operator $T_{0, \Sigma^{ \pm}}$. More precisely, we like to show that the problem is well-posed in a somehow reasonable space setting, and therefore we are looking for a weak solution in the energy space $H^{1}$ such that $g$ has to be (arbitrarily) given in the trace space $H^{1 / 2}\left(\mathbb{R}_{+}\right)=r_{+} H^{1 / 2}$ identifying the two banks $\Sigma^{ \pm}$of the screen with the half-line $\overline{\mathbb{R}_{+}}$. Moreover, an explicit solution in closed analytical form is wanted. See $[77,107]$ for more details.

We shall work mainly with the spaces of Bessel potentials $H^{s}$ which coincide with the Sobolev spaces $W^{s, p}$ in the case $s \in \mathbb{Z}, p=2$ and the Sobolev-Slobodeckij spaces for $s \in$ $\mathbb{R}, p=2$, all briefly referred to as Sobolev spaces [3, 41, 52, 119, 120, 124].

It has been shown [107] that problem (3.21) is solvable if and only if the difference of the traces of the normal derivative of $u$ on the upper and lower bank of the full line $\left\{\left(x_{1}, x_{2}\right) \in\right.$ $\left.\mathbb{R}^{2}: x_{2}=0\right\}$ (identified with $\mathbb{R}$ ), respectively, $f \in H^{-1 / 2}(\mathbb{R})$ satisfies the equation

$$
r_{+} A_{\Phi} f=2 g
$$

where $A_{\Phi}=\mathcal{F}^{-1} \Phi \cdot \mathcal{F}, \Phi(\xi)=\left(\xi^{2}-k^{2}\right)^{-1 / 2}, \xi \in \mathbb{R}$, i.e., $A_{\Phi}$ is a convolution (or pseudodifferential) operator of order -1 and an isomorphism from $H^{-1 / 2}(\mathbb{R})$ onto $H^{1 / 2}(\mathbb{R})$. Furthermore, the functional $f$ has to be supported on the positive half-line, which will be indicated by $f \in H_{+}^{-1 / 2}$ (analogously to the definition of $L_{+}^{2}$ ). It is important that the formulas (2.5) - (2.6) are valid for $H^{s}$ instead of $L^{2}$ if and only if $|s|<1 / 2$, see [41]. The different analytical nature of the spaces $H^{ \pm 1 / 2}\left(\mathbb{R}_{+}\right)$and $H_{+}^{ \pm 1 / 2}$ is crucial. It is well-known $[46,52]$ that the spaces

$$
\widetilde{H}^{s}\left(\mathbb{R}_{+}\right)=\left\{f \in H^{s}\left(\mathbb{R}_{+}\right): r_{+} f \in H_{+}^{s}\right\} \quad, \quad s \geq-\frac{1}{2},
$$


equipped with the norm of $H_{+}^{s}$, satisfy

$$
\begin{aligned}
& \widetilde{H}^{s}\left(\mathbb{R}_{+}\right)=H^{s}\left(\mathbb{R}_{+}\right),|s|<\frac{1}{2}, \\
& \widetilde{H}^{s}\left(\mathbb{R}_{+}\right) \quad \text { dense } \quad H^{s}\left(\mathbb{R}_{+}\right), s= \pm \frac{1}{2}, \\
& \widetilde{H}^{s}\left(\mathbb{R}_{+}\right) \quad \subset \quad C\left(\mathbb{R}_{+}\right), s>\frac{1}{2} .
\end{aligned}
$$

For these reasons it is not convenient to consider equation (3.22) as a WH integral equation of the first kind in $L^{2}$, but as a WH equation in asymmetric space setting with a WHO defined by

$$
W=\left.r_{+} A_{\Phi}\right|_{H_{+}^{-1 / 2}}: H_{+}^{-1 / 2} \rightarrow H^{1 / 2}\left(\mathbb{R}_{+}\right),
$$

where the convolution $A_{\Phi}=\mathcal{F}^{-1} \Phi \cdot \mathcal{F}: H^{-1 / 2} \rightarrow H^{1 / 2}$ is invertible by $A_{\Phi}^{-1}=\mathcal{F}^{-1} \Phi^{-1} \cdot \mathcal{F}$.

Remark 3.4. Note that the operator $W$ does not have the form of a general WHO in asymmetric space setting (2.19), because $r_{+}: H^{1 / 2} \rightarrow H^{1 / 2}\left(\mathbb{R}_{+}\right)$is not a projector in $H^{1 / 2}$, nor is it equivalent to a WHO in $H^{1 / 2}\left(\mathbb{R}_{+}\right)$in the same way as in the $L^{2}$ setting, because $\ell_{0}$ does not act from $H^{1 / 2}\left(\mathbb{R}_{+}\right)$ into $H^{1 / 2}(\mathbb{R})$. However, any continuous extension operator $\ell^{c}: H^{1 / 2}\left(\mathbb{R}_{+}\right) \rightarrow H^{1 / 2}(\mathbb{R})$ with $P_{2}=$ $\ell^{c} r_{+}=P_{2}^{2} \in H^{1 / 2}(\mathbb{R})$ will serve this purpose and leads to more complicated formulas explained below.

The square root function that appears in the Fourier symbol of $A_{\Phi}$ (with $\Im m k>0$ ) is of great importance. We shall denote it by

$$
t(\xi)=\left(\xi^{2}-k^{2}\right)^{1 / 2} \quad, \quad \xi \in \mathbb{R},
$$

and take the branch cut from $k$ to $-k$ vertically via $\infty$ such that

$$
t(\xi) \approx|\xi| \quad, \quad \text { as } \xi \rightarrow \pm \infty .
$$

Its factorisation (with corresponding branches)

$$
t(\xi)=(\xi-k)^{1 / 2}(\xi+k)^{1 / 2}=t_{-}^{1 / 2}(\xi) t_{+}^{1 / 2}(\xi), \quad \xi \in \mathbb{R},
$$

is closely connected with the definition of the one-dimensional Sobolev spaces [41] and some important properties. Firstly, we have

$$
\begin{aligned}
H^{s} & =A_{t^{-s}} L^{2}, \\
H_{+}^{s} & =A_{t_{+}^{-s}} L_{+}^{2}, H_{-}^{s}=A_{t_{-}^{-s}} L_{-}^{2}, \\
H^{s}\left(\mathbb{R}_{+}\right) & =r_{+} A_{t_{-}^{-s}} L_{+}^{2}, H^{s}\left(\mathbb{R}_{-}\right)=r_{-} A_{t_{+}^{-s}} L_{-}^{2},
\end{aligned}
$$

for all $s \in \mathbb{R}$ (in a distributional sense for $s<0$ ). Note that in these formulas the wave number $k$ takes over the common role of the imaginary unit $i$ because of the assumption of $\Im m k>$ 0 (the dependence on $k$ is here suppressed for brevity). Moreover, they serve to construct projectors and extension operators (mentioned before) that are most convenient in the present context $[22,25]$.

To make the connection between (3.23) and (2.19) it is necessary to find convenient projectors and extension operators. This step is a little technical, however leads to a rigorous understanding of classical as general WHOs. 
For any $s \in \mathbb{R}$, we find projectors in $H^{s}$ abbreviating now (for cosmetic reasons) $P_{+}=$ $\ell_{0} r_{+} \in \mathcal{L}\left(L^{2}\right)$ and $P_{-}=I-P_{+}=\ell_{0} r_{-}$by putting

$$
\begin{array}{llll}
P_{+}^{(s)}=A_{t_{+}^{-s}} P_{+} A_{t_{+}^{s}} & \text { onto } H_{+}^{s}, \\
P_{-}^{(s)}=A_{t_{-}^{-s}} P_{-} A_{t_{-}^{s}} & \text { onto } H_{-}^{s}, \\
\Pi_{+}^{(s)}=A_{t_{-}^{-s}} P_{+} A_{t_{-}^{s}} & \text { along } H_{-}^{s}, \\
\Pi_{-}^{(s)}=A_{t_{+}^{-s}} P_{-} A_{t_{+}^{s}} & \text { along } H_{+}^{s},
\end{array}
$$

which are orthogonal (in the common sense) for $k=i$. Hence, $P_{+}^{(s)}+\Pi_{-}^{(s)}=I_{H^{s}}$ and $P_{-}^{(s)}+$ $\Pi_{+}^{(s)}=I_{H^{s}}$ for any $s \in \mathbb{R}$. Suitable extension operators are (because of (3.26)) given by

$$
\begin{aligned}
& \ell_{+}^{(s)}=A_{t_{-}^{-s}} P_{+} A_{t_{-}^{s}} \ell^{(s)} \quad: \quad H^{s}\left(\mathbb{R}_{+}\right) \rightarrow H^{s}(\mathbb{R}), \\
& \ell_{-}^{(s)}=A_{t_{+}^{-s}} P_{-} A_{t_{+}^{s}} \ell^{(s)} \quad: \quad H^{s}\left(\mathbb{R}_{-}\right) \rightarrow H^{s}(\mathbb{R}),
\end{aligned}
$$

where $\ell^{(s)}$ stands for an arbitrary extension into $H^{s}$, see [41]. Obviously, we have the analogue of (2.6)

$$
r_{+} \ell_{+}^{(s)}=I_{H^{s}\left(\mathbb{R}_{+}\right)}, \quad \Pi_{+}^{(s)}=\ell_{+}^{(s)} r_{+} \in \mathcal{L}\left(H^{s}\right) .
$$

Now, we are in the position for a strict understanding of (3.23) in the sense of an asymmetric general $\mathrm{WHO}$ and its inversion in the spirit of Theorem 2.5. First, we see that $W$ is equivalent to the operator

$$
\ell_{+}^{(1 / 2)} W=\left.\Pi_{+}^{(1 / 2)} A_{\Phi}\right|_{\operatorname{im} P_{+}^{(-1 / 2)}}: H_{+}^{-1 / 2} \rightarrow \ell_{+}^{(1 / 2)} H^{1 / 2}\left(\mathbb{R}_{+}\right),
$$

which has the form of (2.19). Second, the idea of a strong factorisation (2.10) (with $C=I$ ) extends to the asymmetric case

$$
\begin{aligned}
A_{\Phi} & =A_{t_{-}^{-1 / 2}} A_{t_{+}^{-1 / 2}} \\
& : H^{1 / 2} \longleftarrow L^{2} \longleftarrow H^{-1 / 2},
\end{aligned}
$$

a factorisation into isomorphisms between the indicated spaces with factor properties analogous to the symmetric space case (2.17)

$$
\begin{aligned}
& P A_{+} P_{1}=A_{+} P_{1}, \quad P_{1} A_{+}^{-1} P=A_{+}^{-1} P, \\
& P_{2} A_{-} P=P_{2} A_{-}, P A_{-}^{-1} P_{2}=P A_{-}^{-1},
\end{aligned}
$$

with the previous interpretation of operators, namely $P_{1}=P_{+}^{(-1 / 2)}, P=\ell_{0} r_{+},, P_{2}=\Pi_{+}^{(1 / 2)}$. It follows that the equation (3.22) is solved by the asymmetric analogue of (2.18):

$$
f=W^{-1} 2 g=\left.A_{+}^{-1} P A_{-}^{-1}\right|_{P_{2} Y} \ell^{(1 / 2)} 2 g .
$$

Moreover, it turns out that the idea of Theorem 2.5 works for general WHOs in asymmetric space setting, as well, see [106, Chapter 2] or [113, Theorem 2.1]:

Theorem 3.3. Let $X, Y$ be Banach spaces, $A \in \mathcal{L}(X, Y)$ an isomorphism and $P_{1} \in \mathcal{L}(X), P_{2} \in$ $\mathcal{L}(Y)$ two projectors. Then the (general) WHO

$$
W=\left.P_{2} A\right|_{P_{1} X} \quad: \quad P_{1} X \rightarrow P_{2} Y
$$


is boundedly invertible if and only if $A$ admits a strong $W H$ factorisation with respect to $\left(X, Y, P_{1}, P_{2}\right)$ :

$$
\begin{aligned}
A & =A_{-} A_{+} \\
& : Y \longleftarrow Z \longleftarrow X,
\end{aligned}
$$

where $Z$ is a Banach space, $P \in \mathcal{L}(Z)$ a projector, and the relations (3.30) are satisfied. In this case, the inverse of $W$ is given by

$$
W^{-1}=\left.A_{+}^{-1} P A_{-}^{-1}\right|_{P_{2} Y} \quad: \quad P_{2} Y \rightarrow P_{1} X .
$$

The previous concept is applicable to various classes of diffraction problems that lead to systems of WH equations, as well. As a first example, we consider the Rawlins problem [77, 93, 107] where, instead of the Dirichlet condition on both banks of the screen (see (3.21)), a Dirichlet condition on the upper bank and a Neumann condition on the lower bank is prescribed, in brief

$$
\begin{aligned}
\left(\Delta+k^{2}\right) u & =0 \text { in } \Omega, \\
T_{0, \Sigma^{+}} u & =\left.u\left(., x_{2}\right)\right|_{x_{2}=+0}=g_{0} \in H^{1 / 2}\left(\mathbb{R}_{+}\right), \\
T_{1, \Sigma^{-}} u & =\partial u /\left.\partial x_{2} u\left(., x_{2}\right)\right|_{x_{2}=-0}=g_{1} \in H^{-1 / 2}\left(\mathbb{R}_{+}\right) .
\end{aligned}
$$

The resulting $2 \times 2$ system of WH equations can be written (after some elementary substitutions) as

$$
r_{+} A_{\Phi} f=g,
$$

where $A$ acts in a topological product (Banach) space (like a $2 \times 2$ matrix operator):

$$
\begin{aligned}
A & =\mathcal{F}^{-1} \Phi \cdot \mathcal{F}: H^{1 / 2} \times H^{-1 / 2} \rightarrow H^{1 / 2} \times H^{-1 / 2}, \\
\Phi & =\left(\begin{array}{cc}
-1 & t^{-1} \\
t & 1
\end{array}\right), \\
f & \in H_{+}^{1 / 2} \times H_{+}^{-1 / 2}, g=\left(g_{0}, g_{1}\right) \in H^{1 / 2}\left(\mathbb{R}_{+}\right) \times H^{-1 / 2}\left(\mathbb{R}_{+}\right) .
\end{aligned}
$$

It turns out that a strong factorisation of $A$ in the form (3.33) can be explicitly obtained with an intermediate space [77]

$$
Z=H^{1 / 4} \times H^{-1 / 4} .
$$

This is an interpretation of the explicit factorisation of the matrix $\Phi$, a spectacular result of Tony Rawlins in 1975 [91, 92], which will be discussed in a wider frame subsequently and leads us to the rigorous solution of a series of boundary-transmission problems and operator theoretical insights, presented below.

Hence, the problem (3.35) is well-posed in this space setting and the solution (resolvent operator) can be obtained by the help of the inversion formula of Theorem 3.2. Moreover, the asymptotical behavior of the solution near the edge of the screen is related to the order of the Sobolev spaces in (3.38):

$$
\operatorname{grad} u \sim|x|^{-3 / 4} \text { as } x \rightarrow 0 .
$$

Further boundary-transmission problems can be studied by analogy when the two boundary conditions in (3.35) are replaced by an arbitrary linear combination of the two Dirichlet data $T_{0, \Sigma^{ \pm}}$in the first place and an arbitrary linear combination of the two Neumann data $T_{1, \Sigma^{ \pm}}$in the second place $[77,108]$ :

$$
\begin{aligned}
\left(\Delta+k^{2}\right) u & =0 \quad \text { in } \Omega \\
a_{0} u_{0}^{+}+b_{0} u_{0}^{-} & =g_{0} \quad, \quad a_{1} u_{1}^{+}+b_{1} u_{1}^{-}=g_{1} \text { on } \Sigma .
\end{aligned}
$$


They lead to a modification in the derived WH equation, namely replacing the Fourier symbol $\Phi$ in (3.37) by

$$
\sigma_{\lambda}=\left(\begin{array}{cc}
1 & t^{-1} \\
t & \lambda
\end{array}\right)
$$

where the parameter $\lambda \in \mathbb{C} \backslash\{0,1\}$ is an algebraic combination of the coefficients $a_{0}, b_{0}, a_{1}, b_{1}$ (under slight conditions on the coefficients that make the boundary operators to be of "normal type", see [108], formulas (1.5), (1.6)). We shall present the explicit factorisation in the next section and a complete discussion of all $\lambda \in \mathbb{C}$ in Section 5, after introducing some auxiliary material.

As a remarkable result the exponent $1 / 4$ in the intermediate space (3.39) has to be replaced by $\delta / 2$, where $\delta \in(0,1]$ is simply computed from $\lambda$ and determines the order of the singularity of $\operatorname{grad} u$ at the origin:

$$
\delta=\Re e\left(\frac{i}{\pi} \log \frac{\sqrt{\lambda}+1}{\sqrt{\lambda}-1}\right) \quad, \quad \operatorname{grad} u \sim|x|^{\delta / 2-1} \text { as } x \rightarrow 0 .
$$

It turns out that, under the assumption $\lambda \notin[1,+\infty)$, all these problems are well-posed in the given setting and explicitly solvable by Theorem 3.2 with the help of a factorisation of the matrix (3.39), see [77, 108] for details.

The same holds true for transmission problems where a pair of functions $u=\left(u^{+}, u^{-}\right)$in the upper and lower half-plane $\Omega^{ \pm}=\left\{x \in \mathbb{R}^{2}: \pm x_{2}>0\right\}$ satisfies

$$
\begin{aligned}
& \left(\Delta+k^{2}\right) u^{ \pm}=0 \quad \text { in } \Omega^{ \pm}, \\
& a_{0} u_{0}^{+}+b_{0} u_{0}^{-}=g_{0}, \quad a_{1} u_{1}^{+}+b_{1} u_{1}^{-}=g_{1} \text { on } \Sigma, \\
& a_{0}^{\prime} u_{0}^{+}+b_{0}^{\prime} u_{0}^{-}=g_{0}^{\prime}, \quad a_{1}^{\prime} u_{1}^{+}+b_{1}^{\prime} u_{1}^{-}=g_{1}^{\prime} \text { on } \Sigma^{\prime},
\end{aligned}
$$

where $u_{0}^{ \pm}, u_{1}^{ \pm}$are the traces of $u$ and its normal derivative, respectively, on $x_{2}= \pm 0$ and the coefficients are any complex numbers.

The Fourier symbol of the derived WH system is in this case given by

$$
\Phi=\frac{1}{a_{0}^{\prime} b_{1}^{\prime}+b_{0}^{\prime} a_{1}^{\prime}}\left(\begin{array}{cc}
a_{0} b_{1}^{\prime}+b_{0} a_{1}^{\prime} & -\left(a_{0} b_{0}^{\prime}+b_{0} a_{0}^{\prime}\right) t^{-1} \\
-\left(a_{1} b_{1}^{\prime}+b_{1} a_{1}^{\prime}\right) t & a_{1} b_{0}^{\prime}+b_{1} a_{0}^{\prime}
\end{array}\right) .
$$

Sommerfeld half-plane problems with first and second kind boundary conditions can be seen as a special case of (3.40) where the third line is replaced by the homogeneous jump conditions

$$
u_{0}^{+}-u_{0}^{-}=0, u_{1}^{+}-u_{1}^{-}=0 \text { on } \Sigma^{\prime} .
$$

Another phenomenon appears when the two boundary conditions (or transmission conditions) have the same order, for instance the two Dirichlet data $T_{0, \Sigma^{ \pm}} u=g^{ \pm} \in H^{1 / 2}\left(\mathbb{R}_{+}\right)$are given $(D D)$ or two Neumann data $T_{1, \Sigma^{ \pm}} u=g^{ \pm} \in H^{-1 / 2}\left(\mathbb{R}_{+}\right)(N N)$ or two impedance data are given (II)

$$
\begin{aligned}
& T_{1, \Sigma^{+}} u+i p^{+} T_{0, \Sigma^{+}} u=g^{+} \in H^{-1 / 2}\left(\mathbb{R}_{+}\right) \\
& T_{1, \Sigma^{-}} u+i p^{-} T_{0, \Sigma^{-}} u=g^{-} \in H^{-1 / 2}\left(\mathbb{R}_{+}\right)
\end{aligned}
$$

with different impedance numbers $p^{ \pm}, \Im m p^{ \pm}>0$, and possibly different data in all cases. Obviously, it is necessary that the difference of the given data $g^{+}-g^{-}$(considered as function(al) on $\mathbb{R}_{+}$) has to be extensible by 0 to $\mathbb{R}_{-}$within the data space $H^{1 / 2}$ or $H^{-1 / 2}$, respectively. 
I.e., the following compatibility conditions are necessary for the solubility of the corresponding problem [76, 108]:

$$
\begin{array}{lll}
g^{+}-g^{-} & \in & \widetilde{H}^{1 / 2}\left(\mathbb{R}_{+}\right) \quad \text { in case } D D, \\
g^{+}-g^{-} & \in & \widetilde{H}^{-1 / 2}\left(\mathbb{R}_{+}\right) \text {in case } N N \text { and } I I .
\end{array}
$$

As a matter of fact these conditions arise automatically from an operator theoretical approach named minimal normalisation of WHOs of the form (2.1) in scales of Sobolev spaces [81, 82, 83].

\section{Historical remarks.}

Sommerfeld diffraction problems are named after Arnold Sommerfeld who found the first closed form solution of a problem of diffraction of a time-harmonic plane wave from a halfplane in terms of Fresnel integrals. It was part of his famous habilitation thesis, published 1896 (in German) in Mathematische Annalen [103, p. 366-371]; see also the annotated translation into English published as a book by R.J. Nagem, M. Zampolli, and G. Sandri [84, Section 8]. The formulation as a boundary value problem goes back to Henri Poincaré [88] who proposed a solution by series expansion in 1892, see [88], [84, p. 121] and [66, p. 145-146].

Several books and survey papers demonstrate the attention on Sommerfeld problems. For an overview about classical WH methods the reader may consult $[2,58,63,71,72,86]$ which report particularly on the pioneering work in diffraction theory by Edward Copson [31], Albert Heins [48, 49], Allan Hurd [54], Douglas Jones [57], Ernst Lüneburg [67], Anthony Rawlins [91, 92, 93], Thomas Senior [97], W.E. Williams [123], and others.

Formulations of Sommerfeld half-plane problems as boundary value and transmission (or interface) problems in Sobolev spaces started in the 1970s with Giorgio Talenti [117], continued in the 1980s by Erhard Meister and his co-authors, touching the more general theory of partial and pseudo-differential equations, for instance by Eli Shamir [99, 100], Mikhail Agranovich, Marko Vishik, and Gregory Eskin [3, 120,41], Joseph Wloka [124], George Hsiao and Wolfgang Wendland [52], to mention some closely related work.

We may point out here particularly the first solutions of the Sommerfeld-Dirichlet and the Sommerfeld-Neumann problems by Edward Copson in 1946 [31] with the classical WH technique and the factorisation of the matrix coming up from the mixed Dirichlet-Neumann problem by Antony Rawlins in 1981 [91, 92, 93]. Erhard Meister and the author started to present resolvent operators in Sobolev spaces for much wider classes of Sommerfeld boundary value and transmission problems in $1985[55,77,107,108,109]$. They also stressed the importance of the intermediate space in operator factorisations, together with Luís Castro [23, 112] and the presence of compatibility conditions together with Francisco Teixeira and Ana Moura Santos $[77,82]$.

The bridge between WHOs and $\Psi$ DOs acting in Sobolev spaces was build by Gregory Eskin in the early 1970s [41]. He also introduced the most convenient notation (3.23) (avoiding the more complicated writing (3.28) presumably for cosmetic reasons) which allowed the consideration of the WHO in a scale of Sobolev spaces, its lifting (cf. Proposition 7.1) etc. The equivalence with operators of the form of a general WHO (2.19) was pointed out by the author in $1983[105,106]$ and systematically used in subsequent publications.

BVPs for systems of PDEs such as the Lamé equations (in crack problems) can be found in $[2,37,78]$ for instance. They are, however, beyond the scope of this article.

\section{CONSTRUCTIVE FACTORISATION OF NON-RATIONAL MATRIX FUNCTIONS}

This topic can be considered as a separate area of research with components in algebra, analysis, matrix and operator theory, which is strongly related to applications. According to the 
width of the area, we confine ourselves to a rough overview after focusing one distinguished class of matrix functions (3.39) as a prototype of those related to the theme of this survey article, which also gave a tremendous impact to factorisation theory [77, 91, 92].

Let us recall the WHO with symbol (3.39) written as

$$
W=\left.r_{+} A\right|_{P_{1} X}: H_{+}^{1 / 2} \times H_{+}^{-1 / 2} \rightarrow H^{1 / 2}\left(\mathbb{R}_{+}\right) \times H^{-1 / 2}\left(\mathbb{R}_{+}\right),
$$

where $X=Y=H^{1 / 2}(\mathbb{R}) \times H^{-1 / 2}(\mathbb{R}), A=\mathcal{F}^{-1} \sigma_{\lambda} \cdot \mathcal{F}$ with

$$
\sigma_{\lambda}=\left(\begin{array}{cc}
1 & t^{-1} \\
t & \lambda
\end{array}\right)
$$

and $t(\xi)=\left(\xi^{2}-k^{2}\right)^{1 / 2}, \xi \in \mathbb{R}, \lambda \in \mathbb{C} \backslash\{0,1\}$. The connection with a general WHO is given by (3.32) where, strictly speaking, $P_{1}=P_{+}^{(1 / 2)} \otimes P_{+}^{(-1 / 2)} \sim \operatorname{diag}\left(P_{+}^{(1 / 2)}, P_{+}^{(-1 / 2)}\right)$ and $P_{2}=$ $\Pi_{+}^{(1 / 2)} \otimes \Pi_{+}^{(-1 / 2)}$. A certain factorisation (with the common holomorphy properties and "low" increase at infinity) is derived with the help of Khrapkov's formulas and Daniele's trick [108]:

$$
\begin{aligned}
\sigma_{\lambda+} & =\left(1-\lambda^{-1}\right)^{-1 / 4}\left(\begin{array}{cc}
c_{+} & -s_{+} \sqrt{\lambda} / t \\
-c_{+} \xi / \sqrt{\lambda}-s_{+} t / \sqrt{\lambda} & s_{+} \xi / t+c_{+}
\end{array}\right), \\
\sigma_{\lambda-} & =\left(1-\lambda^{-1}\right)^{-1 / 4}\left(\begin{array}{cc}
c_{-}-s_{-} \xi / t & -s_{-} \sqrt{\lambda} / t \\
-s_{-} t / \sqrt{\lambda}+c_{-} \xi / \sqrt{\lambda} & c_{-}
\end{array}\right),
\end{aligned}
$$

where

$$
\begin{aligned}
c_{ \pm}(\xi) & =\cosh \left[C \log \gamma_{ \pm}(\xi)\right], \\
s_{ \pm}(\xi) & =\sinh \left[C \log \gamma_{ \pm}(\xi)\right], \\
\gamma_{ \pm}(\xi) & =\frac{\sqrt{k \pm \xi}+i \sqrt{k \mp \xi}}{\sqrt{2 k}}, \xi \in \mathbb{R}, \\
C & =\frac{i}{\pi} \log \frac{\sqrt{\lambda}+1}{\sqrt{\lambda}-1} .
\end{aligned}
$$

Because of the asymptotic behavior of $\sigma_{\lambda \pm}$ at infinity, the corresponding factorisation of $A=$ $\mathcal{F}^{-1} \sigma_{\lambda} \cdot \mathcal{F}$ represents a (so-called strong or canonical) Wiener-Hopf factorisation through a vector Sobolev space, provided $\lambda \notin[1,+\infty)$ :

$$
\begin{gathered}
A_{\lambda} \quad=\quad A_{\lambda-} \quad A_{\lambda+}=\mathcal{F}^{-1} \sigma_{\lambda-} \cdot \mathcal{F} \quad \mathcal{F}^{-1} \sigma_{\lambda+} \cdot \mathcal{F}, \\
H^{1 / 2} \times H^{-1 / 2} \leftarrow Z \leftarrow H^{1 / 2} \times H^{-1 / 2} \\
Z \quad=\quad H^{\vartheta}(\mathbb{R}) \quad, \quad \vartheta=\left(\vartheta_{1}, \vartheta_{2}\right)=\left(\frac{1}{2}(1-\delta), \frac{1}{2}(\delta-1)\right),
\end{gathered}
$$

where $\left.\left.\delta=\Re e C=\frac{-1}{\pi} \arg \frac{\sqrt{\lambda}+1}{\sqrt{\lambda}-1} \in\right] 0,1\right]$. The crucial point is that $\left|\vartheta_{j}\right|<1 / 2$ (cf. [112, Formula (6.4)]), briefly speaking: the Sobolev space orders $\vartheta_{j} \in(-1 / 2,1 / 2)$ where $r_{+}$is a left inverse of $\ell_{0}$.

The asymptotic behavior of the gradient of the solution follows by analogy of the conclusions from (3.38), see [108] for details.

$$
\operatorname{grad} u \sim|x|^{\delta / 2-1} \text { as } x \rightarrow 0 .
$$

The factorisation procedure consists of two steps: First the matrix belongs to the (commutative) so-called Daniele-Krapkov class which admit a meromorphic factorisation within the 
same class in which the two factors have an algebraic behavior at infinity, however is not related to a generalised factorisation in the sense of Simonenko [10,44, 102] and therefore does not directly imply a formula for the inverse of the WHO. The Daniele trick corrects this deficiency by twofold factorisation of a rational matrix function, related to the behavior of the previous at infinity, and a rearrangement of the first factorisation by adding factors in the middle of the first factorisation which compensate the increase [32, 77, 93, 107]. It turns out that the final factorisation can be considered as a factorisation into unbounded factors generating a bounded inverse and as a factorisation into bounded factors in the sense of (3.33), as well, provided $\left|\vartheta_{j}\right|<1 / 2[77,108]$. To understand this technique in detail and to answer the question "What happens in case of $\lambda \in[0,+\infty)$ ?" completely, it is necessary to study the so-called lifting of WHOs in Sobolev spaces to WHOs in $L^{2}$ spaces presented in Section 6. Hence, we come back to this question later.

Now, let us have a wider look at the field of constructive matrix factorisation (related to Sommerfeld problems). The progress in this area is based upon many different ideas. We refer to the books $[30,65]$ and the survey papers $[2,12,40,59,63,95,116,117]$ and point out some basic ideas through relevant keywords that demonstrate the variety of facets in constructive matrix factorisation:

- factorisation of rational matrix functions by means of linear algebra [30],

- commutative matrix factorisation [48, 59],

- the Daniele-Khrapkov class [29, 32, 60],

- the Wiener-Hopf-Hilbert method [53, 54],

- understanding of a matrix factorisation as generalised factorisation in $L^{p}$ spaces [90, 102],

- triangular matrix functions [65, 89],

- asymmetric space settings [106],

- generalised inverses for a unified approach [106],

- lifting [34, 41, 106, 108],

- separation of analytic and algebraic properties [77],

- classification of matrix functions with respect to the number of rationally independent entries $[40,90]$,

- reduction by rational transformation [40],

- meromorphic factorisation [14, 15, 56, 78],

- connection with corona problems and Riemann surfaces [8, 17],

- operator factorisation through an intermediate space $[23,113]$.

Proceeding beyond the classes of matrix functions which appear in Sommerfeld problems to further classes occurring in other canonical diffraction problems such as those for wave guides, wedges etc., one finds other species containing exponential terms, e.g., with an exhausting an still increasing amount of literature, see [8,9], for instance.

Further features such as approximate factorisation and stability questions as well as numerical aspects exceed the scope of this exposition. In the present context, we refer to $[1,14,61,80]$.

\section{Historical remarks.}

This wide field of research developed mainly in the last three decades. We highlight only a few milestones which are most important in the present context. A first basic insight was that matrix functions with rational symbols and those with non-rational symbols are quite different in nature. Rational matrix functions have great importance in system theory [4] and admit a rather complete approach [30]. Non-rational Fourier symbols enter the scene with the square root function appearing in the representation formulas, see Proposition 5.4. Some 
corresponding difficulties in matrix factorisation have been recognized already in the 1950s, see the book of Ben Noble [86].

The importance of the so-called Daniele-Khrapkov class in diffraction theory was recognized by A.A. Khrapkov in 1971. The pioneering results of Anthony Rawlins [92, 93, 94] and Vito Daniele in 1984 [32] released a tremendous investigation of constructive factorisation of matrix functions from this class and its applications, see for instance [16, 40, 63, 74, 77, 90, 95, 109].

The most important lines of research in view of the applications in diffraction theory are connected with the names of Abrahams, Daniele, Heins, Hurd, Jones, Lüneburg, Meister, Mishuris, Noble, Rawlins, Rogosin, Senior, Talenti, and Williams. Related theoretical work (existence questions, functional analytic aspects, etc.) can be found in the work of Böttcher, Câmara, Ehrhardt, Feldman, Gohberg, Krupnik, Litvinchuk, Prössdorf, dos Santos, Silbermann, Simonenko, Spitkovsky, and the author.

See the corresponding references for further studies of the history of matrix factorisation.

\section{OPERATOR FACTORISATION IN BOUNDARY VALUE PROBLEMS}

Now, we demonstrate the connection between the solution of a boundary value problem (BVP) or a transmission problem (TRP) and operator factorisation starting with a quite basic contemplation [110], which leads us to an explicit representation of resolvent operators in the case of so-called canonical diffraction problems. Typically an elliptic linear boundary value problem is written in the form

$$
\begin{aligned}
& A u=f \text { in } \Omega \quad \text { (pde in nice domain) } \\
& B u=g \text { on } \Gamma=\partial \Omega \quad \text { (boundary condition). }
\end{aligned}
$$

I.e., we look for the general solution of the system (5.49), where the following are given: $\Omega$ is a Lipschitz domain in $\mathbb{R}^{n}$ (e.g.), $A \in \mathcal{L}\left(\mathcal{X}, Y_{1}\right), B \in \mathcal{L}\left(\mathcal{X}, Y_{2}\right)$ are bounded linear operators in Banach spaces of function(al)s living on $\Omega$ or $\Gamma=\partial \Omega$, respectively. The data $(f, g)$ are arbitrarily given in a (known product) space $Y=Y_{1} \times Y_{2}$ (denoting the topological product considered as a Banach space).

The situation becomes a bit more transparent if we consider the operator associated with the boundary value problem

$$
L=\left(\begin{array}{c}
A \\
B
\end{array}\right): \mathcal{X} \rightarrow Y=Y_{1} \times Y_{2},
$$

where the data space $Y$ and the solution space $\mathcal{X}$ are usually assumed to be known (eventually modified later for practical reasons and in contrast to free boundary problems or certain inverse problems). It is clear that a linear boundary value problem in the abstract setting (5.49) is wellposed if and only if the operator $L$ is an isomorphism. Thus the main problem is: Find (in a certain form) the inverse (resolvent) of the associated operator $L$ (or a generalised inverse etc.). Associated operators of BVPs were systematically used, e.g., in the work of [13, 27, 28, 38, 39, $41,82,124]$. Linear transmission problems can be considered in the same way, cf. $[107,113]$.

The classical idea to present the possible solution $u \in \mathcal{X}$ by surface and/or volume potentials can be seen as an operator factorisation:

If $\mathcal{K}$ is an isomorphism, then $L$ is equivalently reduced to $T=\mathcal{K} L$ in the sense that the two operators are (algebraically and topologically) equivalent, i.e., that $T$ is representable as

$$
T=E \quad L \quad F,
$$




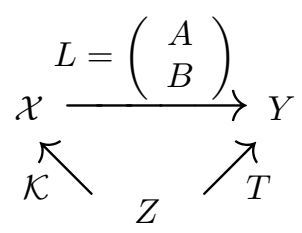

where $E, F$ are linear homeomorphisms. Here $E=\mathcal{K}$ is a potential operator and $F=I, Z=$ $Y$. Equation (5.51) defines an equivalence relation between classes of bounded linear operators in the genuine mathematical sense (reflexive, symmetric and transitive) and practically it includes the idea of a substitution in the solution and in the data space. For the existence of a relation (5.51), we write

$$
T \sim \quad L .
$$

Remark 5.5. In potential theory, the reasoning is often based on a proof that a BVP is uniquely solvable if and only if a system of boundary integral equations is uniquely solvable, see [37, 52]. This would be a consequence of (5.52), however there are other important operator relations which also imply the equivalence of the unique solubility and much more, cf. Section 8.

Let us take a closer look at canonical diffraction problems. There is no strict definition for this kind of boundary value and transmission problems, however experts of the area arrived at a consensus about what it means in conferences $[73,98]$ dedicated to the one hundredth anniversary of Sommerfeld's famous paper [84, 103] as reported in the introduction of [27]: The word "canonical" stands (i) for the particular geometrical situations like axi-parallel, rectangular or circular configurations, (ii) for constant coefficients in the corresponding linear partial differential equations and in the boundary conditions, and (iii) for the importance of the problem to describe basic phenomena in linear time harmonic wave propagation, e.g., governed by the Helmholtz equation.

As a prototype, we focus again the Sommerfeld half-plane problems (3.40) with jump conditions (3.42) on $\Sigma^{\prime}$. Let $\mathcal{H}^{1}(\Omega)$ denote the weak solutions [52] of the Helmholtz equation in the two-dimensional slit domain $\Omega=\mathbb{R}^{2} \backslash \Sigma$ where $\Sigma=\left\{x=\left(x_{1}, x_{2}\right) \in \mathbb{R}^{2}: x_{1} \geq 0, x_{2}=0\right\}$ is the half-line identified with $\mathbb{R}_{+}$as in Section 3. The Sommerfeld half-plane problems with two transmission conditions of first and second kind, respectively, are briefly written as [108]

$$
\begin{aligned}
u & \in \mathcal{H}^{1}(\Omega), \\
B_{1} u & =a_{0} u_{0}^{+}+b_{0} u_{0}^{-}=g_{0} \in H^{1 / 2}\left(\mathbb{R}_{+}\right), \\
B_{2} u & =a_{1} u_{1}^{+}+b_{1} u_{1}^{-}=g_{1} \in H^{-1 / 2}\left(\mathbb{R}_{+}\right) .
\end{aligned}
$$

Remark 5.6. Obviously we have a special case of the BVP (5.49) where $f=0$, hence the associated operator is, instead of (5.50), the following one:

$$
L^{0}=\left.B\right|_{\text {ker } A}: \mathcal{H}^{1}(\Omega) \rightarrow Y_{2}=H^{1 / 2}\left(\mathbb{R}_{+}\right) \times H^{-1 / 2}\left(\mathbb{R}_{+}\right) .
$$

The relation between $L$ and $L^{0}$ will be discussed later in Section 8.

Remark 5.7. Note that we avoided the notation $u \in H^{1}(\Omega)$ here and before in the context of the slit domain $\Omega=\mathbb{R}^{2} \backslash \Sigma$ (in Section 3), because otherwise we had $H^{1}(\Omega)=\left.H^{1}\left(\mathbb{R}^{2}\right)\right|_{\Omega}$ according to the definition of Sobolev spaces which does not allow different traces of $u$ on $\Sigma^{ \pm}$. Hence, $\mathcal{H}^{1}(\Omega)$ strictly speaking denotes functions from $L^{2}(\Omega)$ for which any restriction to a proper sub-cone $\Omega^{\prime} \subset \Omega$ is a $H^{1}\left(\Omega^{\prime}\right)$ solution of the Helmholtz equation in $\Omega^{\prime}$. 
The following representation formula for $u \in \mathcal{H}^{1}(\Omega)$ is well-known $[77,107]$.

Proposition 5.1. A function $u \in L^{2}\left(\mathbb{R}^{2}\right)$ belongs to $\mathcal{H}^{1}(\Omega)$ if and only if

$$
\begin{aligned}
& u^{ \pm}=\left.u\right|_{\Omega^{ \pm}} \in H^{1}\left(\Omega^{ \pm}\right) \text {and hence } u_{0}^{ \pm}=\left.u\right|_{x_{2}= \pm 0} \in H^{1 / 2}(\mathbb{R}), \\
& u_{0}^{+}-u_{0}^{-}=0 \text { and } A_{t}\left(u_{0}^{+}+u_{0}^{-}\right)=0 \text { on } \mathbb{R}_{-}, \\
& u(x)=\mathcal{F}_{\xi \mapsto x_{1}}^{-1} e^{-x_{2} t(\xi)} \widehat{u_{0}^{+}}(\xi) 1_{+}\left(x_{2}\right)+\mathcal{F}_{\xi \mapsto x_{1}}^{-1} e^{x_{2} t(\xi)} \widehat{u_{0}^{-}}(\xi) 1_{-}\left(x_{2}\right), \\
& x=\left(x_{1}, x_{2}\right) \in \Omega^{ \pm},
\end{aligned}
$$

where $u_{0}^{ \pm}$are taken in the sense of the trace theorem, $\widehat{u_{0}^{ \pm}}=\mathcal{F} u_{0}^{ \pm}$, and $1_{ \pm}$stand for the characteristic functions of $\mathbb{R}_{ \pm}$.

We denote the potential operator in (5.55) as $\mathcal{K}$ and write briefly

$$
\begin{aligned}
\mathcal{K} & : H^{1 / 2} \times H^{1 / 2} \rightarrow \mathcal{H}^{1}\left(\Omega^{+}\right) \times \mathcal{H}^{1}\left(\Omega^{-}\right), \\
u & =\mathcal{K}\left(u_{0}^{+}, u_{0}^{-}\right) .
\end{aligned}
$$

It is easily shown that $\mathcal{K}$ is an isomorphism. To solve the problem (5.53), we now determine $\Phi$ from (3.41) - (3.42) (plugging (5.55) into the transmission conditions (5.53)) as

$$
\begin{aligned}
& \Phi=\frac{1}{2}\left(\begin{array}{cc}
a_{0}-b_{0} & -\left(a_{0}+b_{0}\right) t^{-1} \\
-\left(a_{1}+b_{1}\right) t & a_{1}-b_{1}
\end{array}\right) \\
& =\frac{1}{2}\left(\begin{array}{cc}
1 & 0 \\
0 & -\frac{a_{1}+b_{1}}{a_{0}+b_{0}}
\end{array}\right)\left(\begin{array}{cc}
1 & t^{-1} \\
t & \lambda
\end{array}\right)\left(\begin{array}{cc}
a_{0}+b_{0} & 0 \\
0 & -\left(a_{0}+b_{0}\right)
\end{array}\right) \\
& \begin{array}{llll}
E & \sigma_{\lambda} & F
\end{array}
\end{aligned}
$$

with $\lambda=\left(a_{1}-b_{1}\right) /\left(a_{1}+b_{1}\right)$ by elementary transformation with invertible matrices $E, F \in$ $\mathbb{C}^{2 \times 2}$ provided $\left(a_{0} \pm b_{0}\right) \neq 0$ and $\left(a_{1}+b_{1}\right) \neq 0$.

We define $A_{\Phi}=\mathcal{F}^{-1} \Phi \cdot \mathcal{F}: X \rightarrow X X=H^{1 / 2} \times H^{-1 / 2}, P_{1}=P_{+}^{(1 / 2)} \otimes P_{+}^{(-1 / 2)}$, $P_{2}=\Pi_{+}^{(1 / 2)} \otimes \Pi_{+}^{(-1 / 2)}$ (sometimes written as diagonal matrix operators) and carry out the factorisation of $\sigma_{\lambda}$ as given in (4.47) $\sigma_{\lambda}=\sigma_{\lambda-} \sigma_{\lambda+}, A_{\sigma_{\lambda}}=A_{\sigma_{\lambda-}} A_{\sigma_{\lambda+}}$. Further put

$$
W=\left.P_{2} A_{\Phi}\right|_{P_{1} X} \quad, \quad W^{-1}=\left.F^{-1} A_{\sigma_{\lambda+}}^{-1} P A_{\sigma_{\lambda-}}^{-1}\right|_{P_{2} X} E^{-1}
$$

in accordance with Theorem 3.2, where $P=\ell_{0} r_{+}$(cf. also Section 7). The following result is taken from [108].

Theorem 5.4. The BVP (5.53) is well posed, if and only if $\lambda \notin[1,+\infty)$. In this case, the operator $L^{0}$ associated to the (semi-homogeneous) BVP is equivalent to an invertible WHO $W$ and the resolvent operator reads

$$
\left(L^{0}\right)^{-1}=\mathcal{K} W^{-1} .
$$

A complete discussion in dependence of the parameter $\lambda$ will be possible later in Section 7 after equivalent reduction of $W$ to a WHO $W_{0}$ acting in $L^{2}$ spaces, the so-called lifting process.

In brief (operator language) the proof is based upon an equivalence relation

$$
L^{0} \sim W=L^{0} \mathcal{K} \sim W_{0}
$$

and a complete analysis of $W_{0} \in \mathcal{L}\left(L^{2}\left(\mathbb{R}_{+}\right)^{2}\right)$ as a generalisation of the WHO in Section 2 which is only possible after some additional preparation in Section 6. Further concepts of operator factorisation in boundary value problems will be discussed in Section 7. 


\section{Historical remarks.}

In the present sense, operators $L$ associated with elliptic BVPs have been used most consequently in the book of Joseph Wloka [124]. In different context, it appeared already in the area of pseudo-differential operators, see [13] for instance.

The question of whether a BVP is well-posed goes back to Jacques Hadamard (1865-1963), see $[47,68]$. Besides the unique solubility he asked for a continuous dependence of the solution from the data (here from $f$ and $g$ ). Therefore a complete formulation of the linear BVP (5.49) needs at least topological vector spaces (for the sets of data and solutions). The restriction of the consideration to Banach or Hilbert spaces comes from practical reasons and physical motivation (the energy space). See $[52,124]$ for more details.

The formulation of an "equivalent reduction" of a BVP to a boundary integral equation, e.g., has many different forms in the literature, see for instance [52, Section 5.6.5] or [37]. In recent years, it reached a higher level of precision by use of operator relations $[27,77,110,113]$ and became subject of independent research $[4,5,51]$.

\section{ON THE CHOICE OF SYMBOL CLASSES}

In what concerns the classes of WHOs it is quite important and convenient to study not only Fourier symbols which are rational functions or which belong to a decomposing algebra but which are related to a decomposin algebra such as the Wiener algebra or the Banach algebra of Hölder continuous functions on the one-point compactification of the real line $\dot{\mathbb{R}}=\mathbb{R} \cup\{\infty\}$ :

$$
\begin{aligned}
C^{\mu}(\dot{\mathbb{R}})= & \left\{\phi \in C^{\mu}(\mathbb{R}): \phi(\infty)=\lim _{\xi \rightarrow \infty} \phi(\xi)\right. \text { exists and } \\
& \left.\phi(\xi)-\phi(\infty)=\mathcal{O}\left(|\xi|^{-\mu}\right) \text { as } \xi \rightarrow \infty\right\}, \quad \mu \in(0,1) .
\end{aligned}
$$

It is well known $[44,79]$ that $C^{\mu}(\dot{\mathbb{R}})$ forms a unital algebra, which is decomposing, i.e., the Hilbert projectors (also writable with the help of the Hilbert transformation which is here omitted)

$$
\mathcal{F}^{-1} P_{ \pm} \mathcal{F}=\mathcal{F}^{-1} \ell_{0} r_{ \pm} \mathcal{F}=C_{0}^{\mu}(\dot{\mathbb{R}}) \rightarrow C_{0}^{\mu}(\dot{\mathbb{R}})
$$

are continuous where $C_{0}^{\mu}(\dot{\mathbb{R}})=\left\{\phi \in C^{\mu}(\dot{\mathbb{R}}): \phi(\infty)=0\right\}$ and we have an additive decomposition into complemented subspaces

$$
C_{0}^{\mu}(\dot{\mathbb{R}})=C_{+}^{\mu}(\dot{\mathbb{R}}) \oplus C_{-}^{\mu}(\dot{\mathbb{R}}) .
$$

Roughly speaking these are the subspaces of $C_{0}^{\mu}(\dot{\mathbb{R}})$ functions which are holmorphically extensible into the upper and into the lower complex half-plane, respectively, with zero limit at infinity.

The regular functions of the algebra (6.61)

$$
\mathcal{G} C^{\mu}(\dot{\mathbb{R}})=\left\{\phi \in C^{\mu}(\dot{\mathbb{R}}), \phi(\xi) \neq 0 \text { in } \dot{\mathbb{R}}\right\}
$$

form a group which is inverse-closed, i.e., $\phi^{-1} \in \mathcal{G} C^{\mu}(\dot{\mathbb{R}})$ if $\phi \in \mathcal{G} C^{\mu}(\dot{\mathbb{R}})$. These functions allow a factorisation of the form (2.9) (after splitting $\phi(\infty)$ ) within $\mathcal{G} C^{\mu}(\dot{\mathbb{R}})$ with all the consequences (2.10) - (2.15). 
If, instead of the situation before, there are two different limits $\phi( \pm \infty)$ at infinity, we have also a unital algebra denoted by

$$
\begin{aligned}
C^{\mu}(\ddot{\mathbb{R}})= & \left\{\phi \in C^{\mu}(\mathbb{R}): \phi( \pm \infty)=\lim _{\xi \rightarrow \pm \infty} \phi(\xi)\right. \text { exist and } \\
& \left.\phi(\xi)-\phi( \pm \infty)=\mathcal{O}\left(|\xi|^{-\mu}\right) \text { as } \xi \rightarrow \pm \infty\right\}, \quad \mu \in(0,1),
\end{aligned}
$$

which is inverse-closed, as well. Unfortunately $C^{\mu}(\ddot{\mathbb{R}})$ is not decomposing, a factorisation like (2.10) with factors in $C^{\mu}(\ddot{\mathbb{R}})$ does not exist whenever $\phi(-\infty) \neq \phi(+\infty)$.

However, this algebra is crucial in the treatise of canonical diffraction problems since many Fourier symbols $\Phi$ in the related WH equations are generated by polynomials and square root functions like $t^{ \pm 1}, t_{+}^{ \pm 1 / 2}, t_{-}^{ \pm 1 / 2}$, see the examples in Section 3. More precisely, all Fourier symbols that appear in Sommerfeld half plane problems (two-dimensional, only one wave number involved) belong to a certain algebra, the functions generated by polynomials and one square root function $t$ which can be represented by

$$
\Phi=a_{1} Q_{1}+a_{2} Q_{2},
$$

where $a_{j}$ are scalar functions and $Q_{j}$ are rational $2 \times 2$ matrix functions. These facts finally led to the intensive study of the algebra of matrix functions $C^{\mu}(\ddot{\mathbb{R}})^{n \times n}, n \in \mathbb{N}$, particularly in case of $n=1$ and $n=2$ [21, 26, 27, 83].

Let us have a look at the following factorisation theorem for scalar symbols which at the end is no more than a moderate modification of the formulas in Section $2[34,83]$.

Theorem 6.5. Let $\Phi \in \mathcal{G} C^{\mu}(\ddot{\mathbb{R}})$. Then $\Phi$ admits a factorisation

$$
\begin{aligned}
\Phi & =\Phi_{-} \zeta^{\kappa} \Phi_{+} \text {where } \\
\kappa & =\max \left\{z \in \mathbb{Z}: z \leq \Re(\omega)+\frac{1}{2}\right\}, \\
\omega & =\frac{1}{2 \pi i} \int_{\mathbb{R}} d \log \Phi(\xi) d \xi \in \mathbb{C}, \\
\Psi & =\zeta^{-\omega} \Phi^{-1}(+\infty) \Phi, \quad \zeta(\xi)=\frac{\xi-i}{\xi+i}, \quad \xi \in \mathbb{R}, \\
\Psi_{ \pm} & =\exp \left\{\mathcal{F} \ell_{0} r_{ \pm} \mathcal{F}^{-1} \log \Psi\right\} \in C_{ \pm}^{\mu}(\dot{\mathbb{R}}), \\
\Phi_{-} & =\zeta_{-}^{\omega-\kappa} \Psi_{-}, \quad \Phi_{+}=\Phi(+\infty) \zeta_{+}^{\kappa-\omega} \Psi_{+},
\end{aligned}
$$

where $\zeta_{ \pm}(\xi)=\xi \pm i, \xi \in \mathbb{R}$.

Remark 6.8. Note that the complex winding number $\omega=\omega(\Phi)$ of $\Phi$ can be written as $\omega=\kappa+\eta+i \tau$ where $\kappa \in \mathbb{Z}, \eta \in(-1 / 2,+1 / 2], \tau \in \mathbb{R}$. The function $\Psi$ belongs to $C^{\mu}(\dot{\mathbb{R}}), \Psi(\mp \infty)=1$, and $\omega(\Psi)=0$.

Another observation is that $\zeta(\xi)$ may be replaced by $\zeta_{k}(\xi)=(\xi-k) /(\xi+k)=t_{-}(\xi) / t_{+}(\xi)$ which may have advantage in diffraction problems (cf. (7.76)).

Again, we formally define the operator factorisation corresponding with (2.10)

$$
\begin{aligned}
A & =A_{-} C A_{+}, \\
A_{ \pm} & =\mathcal{F}^{-1} \Phi_{ \pm} \cdot \mathcal{F}, \quad C=\mathcal{F}^{-1} \zeta^{\kappa} \cdot \mathcal{F}: L^{2} \rightarrow L^{2} .
\end{aligned}
$$


These formulas admit (at least) two different interpretations. Firstly, (6.67) represents a generalised factorisation in $L^{2}$ in the sense of [102], provided

$$
\Re(\omega)+\frac{1}{2} \notin \mathbb{Z},
$$

see $[23,44,79,83]$. I.e., $t_{+}^{-1} \Phi_{+}^{ \pm 1} \in L^{2}\left(\mathbb{R}_{+}\right), t_{-}^{-1} \Phi_{-}^{ \pm 1} \in L^{2}\left(\mathbb{R}_{-}\right) . A_{ \pm}$in (6.68) must be regarded as unbounded operators in $L^{2}(\mathbb{R})$, densely defined and such that

$$
W^{-}=\left.A_{+}^{-1} P C^{-1} P A_{-}^{-1}\right|_{L_{+}^{2}}
$$

admits a bounded extension by continuity to $\mathcal{L}\left(L^{2}(\mathbb{R})\right)$. Depending on whether $\kappa$ is positive or negative, this formula defines a right or a left inverse of $W=\left.P A\right|_{P X}$ for $X=L^{2}, P=\ell_{0} r_{+}$, and an inverse of $W$ for $\kappa=0$, all this provided $\eta \neq 1 / 2$.

The second interpretation is a factorisation through an intermediate space [112]

$$
\begin{aligned}
A & =A_{-} \quad C \quad A_{+} \\
& : X \longleftarrow Z \longleftarrow Z \longleftarrow
\end{aligned}
$$

where $X=L^{2}$ and $Z=H^{\eta}$ with the advantage to avoid unbounded operators. The consequences are the same as before but formulas are composed of bounded instead of unbounded operators.

In the case of $\eta=1 / 2$ the operator $W$ turns out to be not normally solvable. More precisely, its image is not closed. A normalisation can be achieved systematically by choosing a smaller image space imposing a compatibility condition (in the image space) and a corresponding norm such that the normalised operator is one-sided invertible and (6.70) becomes a one-sided inverse in the new space setting. See [82, 83] for details.

The generalisation of Theorem 6.1 to the systems case where $\Phi$ is a squared matrix function

$$
\Phi \in \mathcal{G} C^{\mu}(\ddot{\mathbb{R}})^{m \times m}
$$

combines ideas of the factorisation of matrix functions with elements in decomposing algebras such as $\Phi \in C^{\mu}(\dot{\mathbb{R}})^{m \times m}$ [43], with the factorisation technique of Theorem 6.1, see [21]. The case $m=2$ is needed for a complete analysis of (5.60).

In the next section, we shall see the abstract analogue of this factorisation (6.71) as a sufficient and necessary condition for the generalised invertibility of a general WHO in symmetric and in asymmetric space setting, respectively.

\section{Historical remarks.}

For a glance at the history of relevant function spaces we refer to the book by Hans Triebel [119]. The use of Sobolev-like spaces in BVPs started in the 1930s with the famous work of Sergei Sobolev, L.N. Slobodeckij, Beppo Levi, and Nachman Aronzjan and became a standard tool in the solubility theory for partial differential and pseudo-differential equations in the 1950s, see the work of Mikhail Agranovic, Nicolas Bourbaki, and Lars Hörmander. For general questions about smoothness and asymptotic behavior of solutions see [3, 120, 41, 52, 124].

The edge and radiation conditions $[96,104]$ are not relevant in the present context, where a solution $u$ denotes a diffracted wave (without sources and sinks) in a suitable (energy) space.

In what concerns the most relevant classes of Fourier symbols we refer to [10, 42, 79]. In the applications the classes of matrix functions with entries from $C^{\mu}(\dot{\mathbb{R}})$ and $C^{\mu}(\ddot{\mathbb{R}})$ play a decisive role, see $[43,62]$ for the origin and $[34,83]$, respectively, for some relevant applications.

The idea of an intermediate space in WH factorisation (related to Sommerfeld problems) arose in [107] and gained further interest in [18, 23, 83, 112]. 


\section{EQUIVALENCE AND REDUCTION VIA OPERATOR FACTORISATION}

To reduce a problem or a system of equations $T f=g$ can often be seen as to find a "simpler operator" $S$ somehow related to the operator $T$ such that conclusions for $S$ imply conclusions for $T$. As before, we focus bounded linear operators in Banach spaces. The easiest case is the situation where the two operators are equivalent, i.e.,

$$
T=E S F,
$$

where $E$ and $F$ are isomorphisms. In this case, many properties of $S$ are transferred to properties of $T$, e.g., invertibility, the Fredholm property, explicit representation of (generalised) inverses, etc. [24] (and vice versa). Conclusions of that kind appeared already in Section 5. So, we shall speak about "operator relations" and their "transfer properties". Now, we outline four further examples of this rather strong equivalence relation which are most important in WHO theory and its applications:

- lifting from Sobolev to Lebesgue spaces [34, 41, 108],

- shifting of WHOs in a scale of Sobolev spaces [83],

- equivalence of a WHO with the truncation of a cross factor $[105,106]$,

- symmetrisation of general WHOs [11].

First, we describe the so-called lifting of WHOs from Sobolev to Lebesgue spaces in the scalar case. In view of (3.23) consider an operator of the form

$$
W=\left.r_{+} A_{\Phi}\right|_{H_{+}^{r}}: H_{+}^{r} \rightarrow H^{s}\left(\mathbb{R}_{+}\right),
$$

where $A_{\Phi}: X=H^{r} \rightarrow Y=H^{s}$ is a bounded convolution operator, i.e., $t^{s-r} \Phi \in L^{\infty}$. Then we have as a consequence of (3.26):

Proposition 7.2. Under these assumptions $W$ is equivalent to a WHO acting in $L^{2}$ spaces:

$$
\begin{aligned}
W \sim W_{0} & =\left.r_{+} A_{\Phi_{0}}\right|_{L_{+}^{2}}: L_{+}^{2} \rightarrow L^{2}\left(\mathbb{R}_{+}\right) \\
\Phi_{0} & =t_{-}^{r} \Phi t_{+}^{-s} .
\end{aligned}
$$

The same idea works perfectly for matrix operators. As an example consider (4.45) again. There we get

$$
\begin{aligned}
W & =\left.r_{+} A_{\sigma_{\lambda}}\right|_{H_{+}^{1 / 2} \times H_{+}^{-1 / 2}}: H_{+}^{1 / 2} \times H_{+}^{-1 / 2} \rightarrow H^{1 / 2}\left(\mathbb{R}_{+}\right) \times H^{-1 / 2}\left(\mathbb{R}_{+}\right) \\
& \sim W_{0}=\left.r_{+} A_{\sigma_{\lambda, 0}}\right|_{L_{+}^{2} \times L_{+}^{2}}: L_{+}^{2} \times L_{+}^{2} \rightarrow L^{2}\left(\mathbb{R}_{+}\right) \times L^{2}\left(\mathbb{R}_{+}\right)
\end{aligned}
$$

with

$$
\begin{aligned}
\sigma_{\lambda, 0} & =\left(\begin{array}{cc}
t_{-}^{1 / 2} & 0 \\
0 & t_{-}^{-1 / 2}
\end{array}\right)\left(\begin{array}{cc}
1 & t^{-1} \\
t & \lambda
\end{array}\right)\left(\begin{array}{cc}
t_{+}^{-1 / 2} & 0 \\
0 & t_{+}^{1 / 2}
\end{array}\right) \\
& =\left(\begin{array}{cc}
\zeta_{k}^{1 / 2} & 1 \\
1 & \lambda \zeta_{k}^{-1 / 2}
\end{array}\right) .
\end{aligned}
$$

This allows a direct application of the theory of systems of WH equations in $L^{2}$ spaces $[43,79]$ and ends up with a complete discussion of properties of $W$ in dependence of the parameter $\lambda$, see [108, Corollary 4.6], as follows:

(1) $\lambda=0: \sigma_{\lambda, 0}$ is triangular. $W_{0}$ decomposes into two single $\mathrm{WH}$ equations that are invertible;

(2) $\lambda=1: \sigma_{\lambda, 0}$ degenerates and $\operatorname{im} W_{0}$ is not closed;

(3) $\lambda \in(0,1): \sigma_{\lambda, 0}$ admits a bounded strong factorisation, $W_{0}$ is invertible; 
(4) $\lambda \in(1, \infty): \sigma_{\lambda, 0}$ admits a function theoretic factorisation but not a generalised factorisation in the $L^{2}$ setting, $\operatorname{im} W_{0}$ is not closed;

(5) $\lambda \notin[0, \infty): \sigma_{\lambda, 0}$ admits an unbounded (generalised) factorisation with vanishing partial indices, $W_{0}$ is invertible.

As a remarkable fact we cannot find any non-invertible Fredholm operator in this class. All operators are either invertible or not normally solvable.

The preceding idea can also be used to discuss regularity properties of (7.75), say, i.e., to look at smoother solutions of the equation $W f=g$ (for smoother data $g$ ). A convenient way is to consider the restricted operator

$$
W_{s}=\left.\operatorname{Rst} r_{+} A_{\Phi_{0}}\right|_{H_{+}^{s}}: H_{+}^{s} \rightarrow H^{s}\left(\mathbb{R}_{+}\right) \quad, \quad \text { for } s>0 .
$$

This makes sense because $H^{s}$ is an invariant subspace of the convolution operator $A_{\Phi_{0}}$. The consideration in larger spaces may be interesting, as well. Hence, we also define

$$
W_{s}=\left.\operatorname{Ext} r_{+} A_{\Phi_{0}}\right|_{H_{+}^{s}}: H_{+}^{s} \rightarrow H^{s}\left(\mathbb{R}_{+}\right) \quad, \quad \text { for } s<0
$$

by continuous extension since $H^{s} \subset L^{2}, H_{+}^{s} \subset L_{+}^{2}$, and $H^{s}\left(\mathbb{R}_{+}\right) \subset L^{2}\left(\mathbb{R}_{+}\right)$are densely embedded for negative $s$. These operators (7.77) and (7.78) are referred to as shifted operators $[83,87]$.

Now, the point is that the shifted operator can also be lifted to the $L^{2}$ level and that lifted shifted WHO has a symbol $\Phi_{s, 0}=t_{-}^{s} \Phi_{0} t_{+}^{-s}=\zeta^{s} \Phi_{0}$ in the scalar case. Hence, if $\Phi_{0}$ admits a factorisation like (6.67), then all lifted shifted Fourier symbols admit a "certain factorisation" of this kind, as well. This technique can be used, e.g., in the cases where the operator $W_{0}$ of (7.76) is not invertible. Namely, $W$ can be normalised by a shift in the scale of Sobolev spaces, i.e., it becomes invertible as an operator restricted like

$$
W^{(\varepsilon)}: H_{+}^{1 / 2+\varepsilon} \times H_{+}^{-1 / 2+\varepsilon} \rightarrow H^{1 / 2+\varepsilon}\left(\mathbb{R}_{+}\right) \times H^{-1 / 2+\varepsilon}\left(\mathbb{R}_{+}\right)
$$

with a suitable $\varepsilon \in(0,1 / 2)$. For more details see [83] and [21] in the matrix case.

For the following let us recall some properties of generalised inverses $T^{-}$of $T \in \mathcal{L}(X, Y)$ as defined already in (2.20). It is well-known that the following assertions are equivalent:

- $T$ is generalised invertible, i.e., $T T^{-} T=T$ for some $T^{-} \in \mathcal{L}(Y, X)$,

- $T$ has a reflexive generalised inverse, i.e., $T^{-} T T^{-}=T^{-}$holds additionally,

- $\operatorname{ker} T$ and $\operatorname{im} T$ are complemented subspaces of $X$ and $Y$, respectively,

- there exist projectors $P_{0} \in \mathcal{L}(X)$ and $P_{1} \in \mathcal{L}(Y)$ onto $\operatorname{ker} T$ and onto im $T$, respectively,

- there exist projectors $P_{0} \in \mathcal{L}(X)$ and $P_{1} \in \mathcal{L}(Y)$ such that, for arbitrary $g \in Y$, the equation $T f=g$ is solvable if and only if $P_{1} g=g$ and, in this case, the general solution of the equation is given by $f=T^{-} g+P_{0} h, h \in X$.

For the proof choose $T^{-} T T^{-}$as a reflexive generalised inverse, and $P_{0}=I-T^{-} T, P_{1}=$ $T T^{-}$in the corresponding places, briefly speaking.

A third example for an equivalent reduction of a WHO by an operator equivalence (7.73) was touched already in (2.10). The following result is just an interpretation of those formulas, extended to the asymmetric case.

Proposition 7.3. Let $W=\left.P_{2} A\right|_{P_{1} X}: P_{1} X \rightarrow P_{2} Y$ be a general WHO with the assumptions of (2.19). Further let

$$
\begin{aligned}
A & =A_{-} \quad C \quad A_{+} \\
& : Y \longleftarrow Z \longleftarrow Z \longleftarrow X,
\end{aligned}
$$


be a factorisation into three isomorphisms with an intermediate Banach space $Z$, and a projector $P \in$ $\mathcal{L}(Z)$ such that

$$
P A_{+} P_{1}=A_{+} P_{1} \quad, \quad P_{2} A_{-} P=P_{2} A_{-} .
$$

Then $W$ satisfies

$$
W=\left.\left.\left.\left.P_{2} A_{-}\right|_{P Z} P C\right|_{P Z} P A_{-}\right|_{P_{1} X} \sim P C\right|_{P Z}
$$

Various examples show that this idea of equivalent reduction yields direct results, provided the operator $C$ has certain properties (as in (2.10)). We call an isomorphism $C \in \mathcal{L}(Z)$ a cross factor with respect to $(Z, P)$ where $P \in \mathcal{L}(Z)$ is a projector if $C^{-1} P C P$ is idempotent, or equivalently if

$$
C^{-1} P C P=P C^{-1} P C P \text {. }
$$

It follows that $C$ splits the space $Z$ twice into four subspaces with

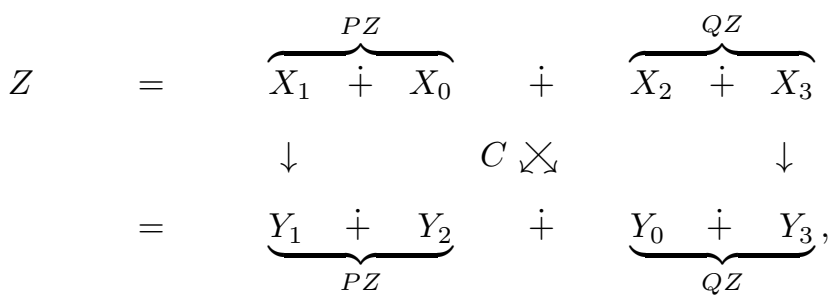

where $Q=I_{Z}-P$ and where $C$ maps each $X_{j}$ onto $Y_{j}, \mathrm{j}=0,1,2,3$, i.e., the complemented subspaces $X_{0}, X_{1}, \ldots, Y_{3}$ are images of corresponding projectors $p_{0}, p_{1}, \ldots, q_{3}$, namely $X_{0}=p_{0} Z=C^{-1} Q C P Z, X_{1}=p_{1} Z=C^{-1} P C P Z, \ldots, Y_{3}=q_{3} Z=C Q C^{-1} Q Z$.

A WHO $W$ which is a truncation of a cross factor, $W=\left.P C\right|_{P X}$, allows very strong conclusions for the solution of the corresponding equation $W f=g$ :

Proposition 7.4. Let $X$ be a Banach space, $P \in \mathcal{L}(X), P^{2}=P$, and $C \in \mathcal{G} \mathcal{L}(X)$ a cross factor with respect to $X, P$. Then we have:

- $W=\left.P C\right|_{P X}$ is generalised invertible,

- $W^{-}=\left.P C^{-1}\right|_{P X}$ is a reflexive generalised inverse of $W$,

- $\operatorname{ker} W=P C^{-1} Q C P X=C^{-1} Q C P X$,

- $\operatorname{im} W=P C^{-1} P C P X=C^{-1} P C P X$,

- for any $g \in P X$ the equation $W f=g$ is solvable in $P X$ if and only if $C^{-1} P C g=g$ and, in this case, the general solution of the equation is given by

$$
f=W^{-} g+C^{-1} Q C h \text { with arbitrary } h \in P X .
$$

The proof of this result is elementary and can be seen as a consequence of the diagram (7.83). Moreover, the equivalence of a general WHO (2.16) in symmetric space setting with $A \in$ $\mathcal{G L}(X)$ to a truncation of a cross factor (in the same space) is characteristic for the generalised invertibility of $W$ :

Theorem 7.6. The WHO (2.16) is generalised invertible if and only if $A$ admits a cross factorisation (with respect to $X$ and $P$ ), i.e.,

$$
A=A_{-} C A_{+},
$$


where $A_{ \pm}$are strong $W H$ factors (satisfying the first two lines of (2.13)) and $C$ is a cross factor (with respect to $X, P)$. In this case, we have

$$
\begin{aligned}
W & =\left.P A_{-} P C P A_{+}\right|_{P X} \\
& =\left.\left.\left.\left.P A_{-}\right|_{P X} P C\right|_{P X} P A_{+}\right|_{P X} \sim P C\right|_{P X}
\end{aligned}
$$

and a reflexive generalised inverse of $W$ is given by

$$
W^{-}=\left.P A_{+}^{-1} P C^{-1} P A_{-}^{-1}\right|_{P X} .
$$

The proof of sufficiency is obvious, a verification of $W^{-}$to be a reflexive generalised inverse based on the factor properties of $A_{ \pm}$and $C$, however not very short. The proof of necessity is tricky, either by use of a space decomposition generated by a reflexive generalised inverse [105] or by a direct formula of a cross factorisation in terms of a generalised inverse, see [106, Chapter 6]. However, a corresponding result for general WHOs in asymmetric space setting $W=\left.P_{2} A\right|_{P_{1} X}$ (see (2.19)) has been published only recently [11, 112], under an additional condition that guarantees the symmetrization of $W$ :

Theorem 7.7. Consider the general WHO (in asymmetric setting) (2.19). Then the following assertions are equivalent:

(1) A admits a WH factorisation through an intermediate space (FIS)

$$
\begin{aligned}
A & =A_{-} \quad C \quad A_{+} \\
& : Y \longleftarrow Z \longleftarrow Z \longleftarrow X,
\end{aligned}
$$

where $A_{ \pm}$are strong $W H$ factors, see (3.30), and $C$ is a cross factor;

(2) $W$ is generalised invertible and $P_{1} \sim P_{2}$, i.e.,

$$
\operatorname{ker} P_{1} \cong \operatorname{ker} P_{2} \text { and } \operatorname{im} P_{1} \cong \operatorname{im} P_{2} .
$$

In this case, a reflexive generalised inverse of $W$ is given by

$$
W^{-}=\left.P_{1} A_{+}^{-1} P C^{-1} P A_{-}^{-1}\right|_{P_{2} Y} .
$$

This last result contains the idea to symmetrise a general WHO and (hence) the space setting itself, see [11] which is important in applications, since it simplifies the reasoning and enables the use of other results, e.g., about operators in Lebesgue spaces (by lifting, as mentioned before). However, the conditions of the last theorem are not necessary for a WHO to be generalised invertible, see the cross factorisation theorem in asymmetric settings in [106] and further going research in [115].

More important for applications is the stability of factorisations against a change of the underlying function spaces for normalisation and regularity results, see [83] for instance.

As an example that fits best to the present context consider the WHO (2.4) again where $C=\mathcal{F}^{-1} \zeta^{\kappa} \cdot \mathcal{F}, \kappa \in \mathbb{Z}$. It is not hard to prove the following facts.

- Formula (2.10) represents a cross factorisation provided $\Phi$ is invertible in the Wiener algebra,

- If $\kappa<0$, the subspaces of (7.83) are

$$
\begin{aligned}
X_{0}=\left.\operatorname{ker} P C\right|_{P X} & =\operatorname{span}\left\{\phi_{j}, j=1, \ldots,-\kappa\right\} \\
\phi_{j}(x) & =\mathcal{F}_{\xi \mapsto x}^{-1} \frac{(\xi-i)^{j-1}}{(\xi+i)^{\kappa}}, x \in \mathbb{R},
\end{aligned}
$$

and $X_{2}$ is not present, etc. 
- If $\kappa>0$, the subspaces of (7.83) are

$$
\begin{aligned}
X_{2}=\left.\operatorname{ker} Q C\right|_{Q X} & =\operatorname{span}\left\{\psi_{j}, j=1, \ldots, \kappa\right\} \\
\psi_{j}(x) & =\mathcal{F}_{\xi \mapsto x}^{-1} \frac{(\xi+i)^{j-1}}{(\xi-i)^{\kappa}}, x \in \mathbb{R},
\end{aligned}
$$

and $X_{0}$ is not present, etc.

In the first case, the equation $W f=g$ is solvable for all $g \in L^{2}\left(\mathbb{R}_{+}\right)$by

$$
f=W^{-} g+\sum_{j=1}^{-\kappa} a_{j} A_{+}^{-1} \phi_{j} \text { with } a_{j} \in \mathbb{C} .
$$

In the second case, the equation $W f=g$ is solvable if and only if $\kappa$ conditions are satisfied

$$
\int_{\mathbb{R}_{+}} g P A_{-}^{-1} P C^{-1} \psi_{j}=0 \quad, \quad j=1, \ldots, \kappa .
$$

The solution reads $f=W^{-} g$ and is unique in this case.

These results are standard conclusions for Fredholm operators and their generalised inverses.

Finally, we like to remark that, in a symmetric space setting, every general WHO $W=$ $\left.P A\right|_{P X}$ which is generalised invertible can be presented as a truncation of a (suitable) cross factor [115]. This underlines the impression that cross factors are something natural, if we look for non-invertible Fredholm or other generalised invertible operators, see [112] for further examples.

\section{Historical remarks.}

The idea of equivalent reduction in the sense of (7.73) is a genuine element of WH factorisation and just an interpretation of the pioneering work mentioned in Section 2. The idea of the lifting process and its consequences was settled by Gregory Eskin and Roland Duduchava in the 1970 s, see [34, 41].

For the history of generalised inverses see [85]. The generalised inversion of general WHOs was firstly studied by the author in [105, 106], where also the term "cross factor" was introduced (in symmetric and in asymmetric Banach space settings). Intermediate spaces in the context of generalised factorisation of matrix functions in the sense of I.B. Simonenko [102] were firstly studied by Luís Castro and the author $[18,23]$. The complete proof of Theorem 7.5 was presented only in 2016 [11, 112].

The question whether a general WHO (2.19) in asymmetric setting can be equivalently reduced to a WHO (2.16) in symmetric setting was recently analysed in [11]. Further questions about equivalent reduction of general WHOs to more convenient forms have been answered in [115].

\section{FROM OPERATOR FACTORISATION TO OPERATOR RELATIONS}

The operator factorisations considered before can all be seen as equivalence relations in the sense of (7.73). However, there are other operator relations which play a crucial role in the present context and in general, as well. We point out two of them, again coming up from concrete applications.

It is well known that the diffraction problem where the trace of the diffracted field is given on a plane screen $\Sigma \in \mathbb{R}^{3}$ (equally on the two sides of the screen) leads to an (boundary integral) 
equation

$$
r_{\Sigma} A_{t^{-1}} f=g
$$

where $\Sigma$ is identified with a (Lipschitz) domain in $\mathbb{R}^{2}, t\left(\xi_{1}, \xi_{2}\right)=\left(\xi_{1}^{2}+\xi_{2}^{2}-k^{2}\right)^{1 / 2}, A_{t^{-1}}=$ $\mathcal{F}^{-1} t^{-1} \cdot \mathcal{F}, \mathcal{F}$ denotes here the two-dimensional Fourier transformation, $g \in H^{1 / 2}(\Sigma)$ is given and $f \in H_{\Sigma}^{-1 / 2}$ is unknown, in analogy to the Sommerfeld-Dirichlet problem: $f$ is an element of $H^{-1 / 2}\left(\mathbb{R}^{2}\right)$ supported on $\bar{\Sigma}$.

Assuming that the complementary screen $\Sigma^{\prime}=\mathbb{R}^{2} \backslash \bar{\Sigma}$ is also a Lipschitz domain, we consider the Sommerfeld-Neumann problem for $\Sigma^{\prime}$ and arrive by analogy at an equation

$$
r_{\Sigma^{\prime}} A_{t} f_{*}=g_{*},
$$

where $g_{*} \in H^{-1 / 2}\left(\Sigma^{\prime}\right)$ is given and $f_{*} \in H_{\Sigma^{\prime}}^{1 / 2}$ is unknown. Now, Lipschitz domains allow continuous extension operators [124], say

$$
\ell_{\Sigma}^{(1 / 2)}: H^{1 / 2}(\Sigma) \rightarrow H^{1 / 2}\left(\mathbb{R}^{2}\right) \quad, \quad \ell_{\Sigma^{\prime}}^{(-1 / 2)}: H^{-1 / 2}\left(\Sigma^{\prime}\right) \rightarrow H^{-1 / 2}\left(\mathbb{R}^{2}\right)
$$

such that the projector $P_{2}=\ell_{\Sigma}^{(1 / 2)} r_{\Sigma}$ acts in $H^{1 / 2}\left(\mathbb{R}^{2}\right)$ along $H_{\Sigma^{\prime}}^{1 / 2}$ and $Q_{1}=\ell_{\Sigma^{\prime}}^{(-1 / 2)} r_{\Sigma^{\prime}}$ acts in $H^{-1 / 2}\left(\mathbb{R}^{2}\right)$ along $H_{\Sigma}^{-1 / 2}$. In analogy to the half-line case in Section 3 , we conclude that the operators in the foregoing equations (8.95) and (8.96) are equivalent to operators in the form of general WHOs

$$
\begin{aligned}
\left.r_{\Sigma} A_{t^{-1}}\right|_{H_{\Sigma}^{-1 / 2}} & \sim W=\left.P_{2} A_{t^{-1}}\right|_{P_{1} X}, \\
\left.r_{\Sigma^{\prime}} A_{t}\right|_{H_{\Sigma^{\prime}}^{1 / 2}} & \sim W_{*}=\left.Q_{1} A_{t}\right|_{Q_{2} Y},
\end{aligned}
$$

where $X=H^{-1 / 2}, P_{1}+Q_{1}=\left.I\right|_{H^{-1 / 2}}$ and $Y=H^{1 / 2}, P_{2}+Q_{2}=\left.I\right|_{H^{1 / 2} \text {, cf. (2.19). Using }}$ the space decompositions $X=P_{1} X \oplus Q_{1} X \cong P_{1} X \times Q_{1} X$ (identifying the direct sum with the topological product space considered as a Banach space written in matrix form) and $Y=$ $P_{2} Y \oplus Q_{2} Y \cong P_{2} Y \times Q_{2} Y$, as well, we have

$$
A_{t^{-1}} \sim\left(\begin{array}{cc}
W & \cdot \\
\cdot & \cdot
\end{array}\right)=\left(\begin{array}{cc}
\cdot & \cdot \\
\cdot & W_{*}
\end{array}\right)^{-1} \sim A_{t}^{-1},
$$

where the dots stand for obvious entries $\left.P_{2} A_{t^{-1}}\right|_{Q_{1} X}$ etc., i.e., $W$ and $W_{*}$ are matricially coupled (MC) operators [4,5] and associated WHOs in the sense of [105, 106].

The MC relation between two bounded linear operators $T, S$ in Banach spaces is often written in the form

$$
\left(\begin{array}{ll}
T & \cdot \\
\cdot & \cdot
\end{array}\right)=\left(\begin{array}{ll}
\cdot & \cdot \\
\cdot & S
\end{array}\right)^{-1} .
$$

It is well known that (8.99) is closely related to another operator relation which is called equivalence after extension (EAE) relation defined by

$$
\left(\begin{array}{cc}
T & 0 \\
0 & I_{Z_{1}}
\end{array}\right)=E\left(\begin{array}{cc}
S & 0 \\
0 & I_{Z_{2}}
\end{array}\right) F,
$$

where $Z_{1}, Z_{2}$ are Banach spaces and $E, F$ are isomorphisms acting between the corresponding spaces. In this case, one writes

$$
T \stackrel{*}{\sim} S .
$$

The two relations (8.99) and (8.100) look quite different, however it turns out that they coincide: 
Theorem 8.8 (of Bart and Tsekanovskii). Two bounded linear operators acting in Banach spaces are matricially coupled if and only if they are equivalent after extension.

There are some important facts to be pointed out:

- EAE is an equivalence relation in the genuine mathematical sense (reflexive, symmetric, and transitive) and such is $\mathrm{MC}$;

- $T \sim S$ implies $T \stackrel{*}{\sim} S$, but not conversely (see the example below);

- The two relations (8.99) and (8.100) can be computed from each other [5, 114].

The following conclusion is most important in applications [24, Theorem 2.5].

Theorem 8.9. If (8.100) is satisfied, and if $S^{-}$is a reflexive generalised inverse of $S$, then a reflexive generalised inverse of $T$ is given by the formula

$$
T^{-}=R_{11}\left(F^{-1}\left(\begin{array}{cc}
S^{-} & 0 \\
0 & I_{Z_{2}}
\end{array}\right) E^{-1}\right),
$$

where $R_{11}$ denotes the restricted operator of the first matrix entry to the first component spaces.

An example for the ease of a MC relation appears in diffraction theory in the context of diffraction of time-harmonic waves from plane screens in $\mathbb{R}^{3}$. It turns out that the operators associated to certain problems for complementary plane screens are matricially coupled, which was denoted as "abstract Babinet principle" in [111]. Therefore, the construction of the resolvent operator for one of the problems implies a representation of the resolvent operator for the other one. Since the resolvent operators can be computed for certain convex screens, the preceeding idea yields the explicit solution of diffraction problems for a much wider class of plane screens, the so-called "polygonal-conical screens" [22].

Another application of the EAE relation is the following. A process commonly denoted by "equivalent reduction" is the step from a boundary value problem (BVP) to a semi-homogeneous BVP. This is not reflected by equivalence between the two associated operators but by equivalence after extension.

Consider the abstract BVP (5.1)-(5.2) and its associated operator (5.50). Moreover consider the semi-homogeneous (abstract) boundary value problem briefly written as

$$
L^{0} u=\left(\begin{array}{c}
A \\
B
\end{array}\right) u=\left(\begin{array}{c}
0 \\
g
\end{array}\right) \in\{0\} \times Y_{2} \cong Y_{2}
$$

with associated operator

$$
\left.B\right|_{\text {ker } A}: X_{0}=\operatorname{ker} A \rightarrow Y_{2} \text {. }
$$

By analogy, we may define $\left.A\right|_{\text {ker } B}: \operatorname{ker} B \rightarrow Y_{1}$.

Theorem 8.10. Let $L=\left(\begin{array}{c}A \\ B\end{array}\right) \in \mathcal{L}\left(\mathcal{X}, Y_{1} \times Y_{2}\right)$ be a bounded linear operator in Banach spaces. Then

$$
\begin{array}{lll}
A R=I \text { for some } R \in \mathcal{L}\left(Y_{1}, \mathcal{X}\right) & \text { implies } & \left.L \stackrel{*}{\sim} B\right|_{\text {ker } A}, \\
B R=I \text { for some } R \in \mathcal{L}\left(Y_{2}, \mathcal{X}\right) & \text { implies } & \left.L \stackrel{*}{\sim} A\right|_{\text {ker } B} .
\end{array}
$$

The proof (for the first case) is based upon the operator identity

$$
L=E T F=\left(\begin{array}{cc}
0 & \left.A\right|_{X_{1}} \\
\left.I\right|_{Y_{2}} & \left.B\right|_{X_{1}}
\end{array}\right)\left(\begin{array}{cc}
\left.B\right|_{X_{0}} & 0 \\
0 & \left.I\right|_{X_{1}}
\end{array}\right)\left(\begin{array}{c}
P \\
Q
\end{array}\right)
$$

which holds if $A$ is right invertible: $A R=\left.I\right|_{Y_{1}}$, say, with $X_{0}=\operatorname{ker} A, X_{1}=\operatorname{im} R, Q=R A, P=$ $I-R A$. Formula (8.105) is an EAE relation between $L$ and $\left.B\right|_{X_{0}}$, more precisely an equivalence 
after one-sided extension (EAOE) relation, since the operator $L$ on the left side is not extended. For details and further consequences (transfer properties, normalization etc.) see [110]. As a consequence, the resolvent operator $\left(L^{0}\right)^{-1}$ of the semi-homogeneous BVP (8.103) implies a representation of the resolvent operator $L^{-1}$ of the full problem by the help of formula (8.102).

For further operator relations, their interplay, and further applications see $[6,19,24,50,51$, 118].

\section{Historical remarks.}

The present concepts of equivalence and reduction are much stronger than those usually used in potential theory where, for instance elliptic BVPs are said to be equivalent to certain boundary integral equations if, roughly speaking, there are mappings which generate solutions from each other, see [52, Section 5.6.5] and [77, Section 2], for instance. Various other equivalence relations appear in the literature, e.g., defined by the fact that two operators are simultaneously Fredholm or not [7]. Those properties can be regarded as consequences of the MC and EAE relations and were denoted as "transfer properties" in [110,115].

The MC and EAE relations were introduced by Harm Bart, Israel Gohberg, and Rien Kaashoek in the early 1980s [4], where the conclusion from MC to EAE was of great importance in system theory, see also [114]. The proof of the inverse conclusion (from EAE to MC) was obtained by Harm Bart and V.E. Tsekanovskii [5]. It gave a great impact to the study of operator relations in general, see [50,51, 110,118]. Its usefulness in the study of various classes of operators was demonstrated already by Luís Castro and the author in 1998 [19, 24, 25] and recently also for applications in diffraction theory, together with Roland Duduchava [22, 111, 113].

\section{THE STEP FROM 2 TO 3 AND TO $n$ DIMENSIONS}

In this final section, we describe briefly an idea of how to study the Sommerfeld problems of Section 3 in higher dimensions, i.e., where $\Omega$ is three-dimensional or even n-dimensional which mathematically does not make a great difference to some extend. The FIS concept (see Theorem 7.5) improves to be very efficient, because the intermediate space turns out to be an anisotropic Sobolev space and the reasoning of an operator factorisation with bounded instead of unbounded operators applies as before in two dimensions.

Instead of a half-line (as in Section 3) consider the half-plane $\Sigma=\mathbb{R}_{1+}^{2}=\left\{x \in \mathbb{R}^{2}: x_{1}>0\right\}$, the (orthogonal) projectors $P_{+}=\ell_{0} r_{+}: L^{2}\left(\mathbb{R}^{2}\right) \rightarrow L^{2}\left(\mathbb{R}^{2}\right)$ onto $L_{\Sigma}^{2}=L_{\mathbb{R}_{1+}^{2}}^{2}, P_{-}=I-P_{+}$and the Bessel potential operators $[35,36,41]$ of order $s \in \mathbb{R}$ :

$$
\begin{array}{ll}
\Lambda_{+}^{s}=A_{\lambda_{+}^{s}}=\mathcal{F}^{-1} \lambda_{+}^{s} \cdot \mathcal{F}, \quad \lambda_{+}^{s}(\xi)=\left(\xi_{1}+i \sqrt{\xi_{2}^{2}+1}\right)^{s}, \xi \in \mathbb{R}^{2} \\
\Lambda_{-}^{s}=A_{\lambda_{-}}=\mathcal{F}^{-1} \lambda_{-}^{s} \cdot \mathcal{F} \quad, \quad \lambda_{-}^{s}(\xi)=\left(\xi_{1}-i \sqrt{\xi_{2}^{2}+1}\right)^{s}, \xi \in \mathbb{R}^{2},
\end{array}
$$

where $\mathcal{F}$ now denotes the two-dimensional Fourier transformation. For any $s \in \mathbb{R}$, we find the orthogonal projectors [25]

$$
\begin{aligned}
& P_{+}^{(s)}=\Lambda_{+}^{-s} P_{+} \Lambda_{+}^{s} \text { onto } H_{\Sigma}^{s} \\
& P_{-}^{(s)}=\Lambda_{-}^{-s} P_{-} \Lambda_{-}^{s} \text { onto } H_{\Sigma^{\prime}}^{s} \\
& \Pi_{+}^{(s)}=\Lambda_{-}^{-s} P_{+} \Lambda_{-}^{s} \text { along } H_{\Sigma^{\prime}}^{s} \\
& \Pi_{-}^{(s)}=\Lambda_{+}^{-s} P_{-} \Lambda_{+}^{s} \text { along } H_{\Sigma}^{s} .
\end{aligned}
$$

Hence $P_{+}^{(s)}+\Pi_{-}^{(s)}=I_{H^{s}}$ and $P_{-}^{(s)}+\Pi_{+}^{(s)}=I_{H^{s}}$. 
As mentioned in Remark 6.2 one can work with $\sqrt{\xi_{2}^{2}-k^{2}}$ instead of $\sqrt{\xi_{2}^{2}+1}$. In that case, the corresponding projectors are not anymore orthogonal in the usual sense.

Now, let us go to the higher-dimensional case $(m=n-1 \geq 2)$ where $\Sigma$ is a half-space, cf. $[20,41,45,100,106]$. According to the traditional notation [41] $x_{n}$ denotes now the "normal derivative direction" (corresponding to $x_{2}$ in Section 3) and $x_{m}=x_{n-1}$ denotes the "WH direction" (corresponding to $x_{1}$ in Section 3 ) whilst the remaining variables $x_{1}, \ldots, x_{m-1}$ play the role of parameters. Putting

$$
\left.X=Y=H^{1 / 2}\left(\mathbb{R}^{m}\right) \times H^{-1 / 2}\left(\mathbb{R}^{m}\right), \quad \Sigma=\mathbb{R}_{+}^{m}=\mathbb{R}^{m-1} \times\right] 0, \infty[
$$

and $t(\xi)=\left(\xi_{1}^{2}+\ldots+\xi_{m}^{2}-k^{2}\right)^{1 / 2}, \xi=\left(\xi^{\prime}, \xi_{m}\right) \in \mathbb{R}^{m}$, we can consider the same factorisation given by (4.47) replacing $k$ by $\left(k^{2}-\xi^{2}\right)^{1 / 2}$, i.e., the previous factorisation as to be parameterdependent of $\xi^{\prime} \in \mathbb{R}^{m-1}$. It turns out that the factorisation (4.47) can be seen as a canonical FIS of $A$ with an intermediate space which is an anisotropic vector Sobolev space

$$
\begin{aligned}
Z & =H^{\vartheta}\left(\mathbb{R}^{m}\right) \times H^{-\vartheta}\left(\mathbb{R}^{m}\right), \\
H^{\vartheta}\left(\mathbb{R}^{m}\right) & \left.\left.=\mathcal{F}\left(w_{\vartheta} L^{2}\left(\mathbb{R}^{m}\right)\right) \quad, \quad w_{\vartheta}(\xi)=\left(1+\left|\xi^{\prime}\right|^{2}\right)^{\vartheta_{1} / 2}\right)\left(1+\xi_{m}^{2}\right)^{\vartheta_{2} / 2}\right), \\
\vartheta & =\left(\vartheta_{1}, \vartheta_{2}\right)=\left(\frac{1}{2}(\delta-1), \frac{1}{2}(1-\delta)\right),
\end{aligned}
$$

see [113] for more details. Adding in the factorisation $\sigma_{\lambda}=\sigma_{\lambda-} \sigma_{\lambda+}$ (cf. (4.47)) a middle factor of the form $\operatorname{diag}\left(\zeta^{\kappa_{1}}, \zeta^{\kappa_{2}}\right)$, where $\zeta(\xi)=\left(\xi_{m}-i\left|\xi^{\prime}\right|\right) /\left(\xi_{m}+i\left|\xi^{\prime}\right|\right)$, we find further examples with non-canonical FIS which are not Fredholm according to a non-trivial kernel (or co-kernel) that is translation invariant with respect to $x^{\prime}=\left(x_{1}, \ldots, x_{m-1}\right) \in \mathbb{R}^{m-1}$, hence infinite dimensional. However, generalised inverses can be constructed as before according to the FIS interpretation.

One can find plenty of further boundary value and transmission problems in higher dimensions $(n \geq 3)$, where the associated operators are not anymore Fredholm but generalised invertible, just adding one or more variables, see [28, 64, 81, 113], for instance.

Many problems in mathematical physics, for instance in elasticity theory, make sense only in three dimensions. They typically lead us to Wiener-Hopf equations, anisotropic function spaces and normalisation problems, see [37, 52, 78, 109], e.g.

Conclusion. The operator theoretical formulation allows a clear and compact description of results about the solution of linear BVPs, a simultaneous treatise of large classes of problems, and the solution of new classes of BVPs, as well.

For instance, the described ideas are applicable to other geometrical situations, particularly the conception of equivalent reduction via operator relations, see further work on diffraction by wedges or polygonal-conical screens etc. such as [20, 22] and [28].

Acknowledgment. The work was supported by FCT - Portuguese Foundation for Science and Technology through the Center for Functional Analysis, Linear Structures and Applications at Instituto Superior Técnico, Universidade de Lisboa, and by a voluntary agreement of the author with the Universidade de Lisboa. The author likes to thank the organisers of the scientific event "Bringing pure and applied analysis together via the Wiener-Hopf technique, its generalisations and applications", at Isaac Newton Institute for Mathematical Sciences, University of Cambridge, England, for their invitation to present the paper in the summer school in August 2019 and for related financial support. 


\section{REFERENCES}

[1] I. D. Abrahams: The application of Padé approximants to Wiener-Hopf factorization. IMA J. Appl. Math. 65 (2000), 257-281.

[2] I. D. Abrahams: On the application of the Wiener-Hopf technique to problems in dynamic elasticity. Wave Motion 36 (2002), 311-333.

[3] M. S. Agranovich, M. I. Vishik: Elliptic problems with a parameter and parabolic problems of general type. Russian Math. Surveys 19 (1964), 53-157. Translation from Usp. Mat. Nauk 19 (1964), 53-161, Russian.

[4] H. Bart, I. Gohberg and M. Kaashoek: The coupling method for solving integral equations. In: Topics in operator theory systems and networks, Workshop Rehovot/Isr. (1983), Oper. Theory Adv. Appl. 2, Birkhäuser, Basel (1984), 39-73. Addendum in Integr. Equ. Op. Theory 8 (1985), 890-891.

[5] H. Bart, V. E. Tsekanovskii: Matrical coupling and equivalence after extension. In: Operator Theory and Complex Analysis, Eds: T. Ando et al., Oper. Theory Adv. Appl. 59, Birkhäuser, Basel (1991), 143-160.

[6] H. Bart, V. E. Tsekanovskii: Complementary Schur complements. Linear Algebra Appl. 197 (1994), 651-658.

[7] M.A. Bastos, A.F. dos Santos and R. Duduchava: Finite interval convolution operators on the Bessel potential spaces $H_{p}^{s}$. Math. Nachr. 173 (1995), 49-63.

[8] M. A. Bastos, Yu. I. Karlovich, A. F. dos Santos and P. M. Tishin: The corona theorem and the canonical factorization of triangular AP matrix functions - Effective criteria and explicit formulas. J. Math. Anal. Appl. 223 (1998), 523-550.

[9] A. Böttcher, Yu. I. Karlovich and I. M. Spitkovsky: Convolution Operators and Factorization of Almost Periodic Matrix Functions. Oper. Theory Adv. Appl. 131, Birkhäuser, Basel (2002).

[10] A. Böttcher, B. Silbermann: Analysis of Toeplitz Operators. Springer, Berlin (2006).

[11] A. Böttcher, F. -O. Speck : On the symmetrization of general Wiener-Hopf operators. J. Operator Theory 76 (2016), 335-349.

[12] A. Böttcher, I. M. Spitkovsky: The factorization problem: some known results and open questions., In: Operator Theory, Operator Algebras and Applications Eds: A. Almeida et al., Oper. Theory Adv. Appl. 229, Birkhäuser, Basel (2013), 101-122.

[13] L. Boutet de Monvel: Boundary problems for pseudo-differential operators. Acta Math. 126 (1971), 11-51.

[14] M. C. Câmara, A. B. Lebre and F. -O. Speck: Meromorphic factorization, partial index estimates and elastodynamic diffraction problems. Math. Nachr. 157 (1992), 291-317.

[15] M. C. Câmara, M. T. Malheiro: Meromorphic factorization revisited and application to some groups of matrix functions. Complex Anal. Oper. Theory 2 (2008), 299-326.

[16] M. C. Câmara, A. F. dos Santos and M. A. Bastos: generalized factorization for Daniele-Khrapkov matrix functions explicit formulas. J. Math. Anal. Appl. 190 (1995), 295-328.

[17] M. C. Câmara, A. F. dos Santos and P. F. dos Santos: Matrix Riemann-Hilbert problems and factorization on Riemann surfaces. J. Funct. Anal. 255 (2008), 228-254.

[18] L. P. Castro: The Characterization of the Intermediate Space in generalized Factorizations. MSc thesis, Universidade Técnica de Lisboa (1994), vii + 90 p., Portuguese.

[19] L. P. Castro: Relations between Singular Operators and Applications. PhD thesis, Universidade Técnica de Lisboa (1998), xix $+163 \mathrm{p}$.

[20] L. P. Castro, R. Duduchava and F. -O. Speck: Localization and minimal normalization of some basic mixed boundary value problems. In: Factorization, Singular Operators and Related Problems, Eds: S. Samko et al., Kluwer, Dordrecht (2003), 73-100.

[21] L. P. Castro, R. Duduchava and F. -O. Speck: Asymmetric factorizations of matrix functions on the real line. In: Modern Operator Theory and Applications. The Igor Borisovich Simonenko Anniversary Volume Eds: Y.M. Erusalimskii et al., Oper. Theory Adv. Appl. 170, Birkhäuser, Basel (2006), 53-74.

[22] L. P. Castro, R. Duduchava and F. -O. Speck: Diffraction from polygonal-conical screens - an operator approach. In: Operator Theory, Operator Algebras and Applications Eds: A. Bastos et al., Oper. Theory Adv. Appl. 242, Birkhäuser, Basel (2014), 113-137.

[23] L. P. Castro, F. -O. Speck: On the characterization of the intermediate space in generalized factorizations. Math. Nachr. 176 (1995), 39-54.

[24] L. P. Castro, F. -O. Speck: Regularity properties and generalized inverses of delta-related operators. Z. Anal. Anwend. 17 (1998), 577-598.

[25] L. P. Castro, F. -O. Speck: Relations between convolution type operators on intervals and on the half-line. Integral Equations Oper. Theory 37 (2000), 169-207.

[26] L. P. Castro, F. -O. Speck: Inversion of matrix convolution type operators with symmetry. Port. Math. (N.S.) 62 (2005), 193-216. 
[27] L. P. Castro, F. -O. Speck and F.S. Teixeira: On a class of wedge diffraction problems posted by Erhard Meister. In: Operator theoretical methods and applications to mathematical physics. The Erhard Meister memorial volume Eds. I. Gohberg et al., Oper. Theory Adv. Appl. 147 (2004), 213-240.

[28] L. P. Castro, F. -O. Speck and F.S. Teixeira: Mixed boundary value problems for the Helmholtz equation in a quadrant. Integr. Equ. Oper. Theory 56 (2006), 1-44.

[29] G. N. Chebotarev: Several remarks on the factorization of operators in a Banach space and the abstract Wiener-Hopf equation. Mat. Issled. 2 (1968), 215-218, Russian.

[30] K. F. Clancey, I. Gohberg : Factorization of Matrix Functions and Singular Integral Operators. Oper. Theory Adv. Appl. 3, Birkhäuser, Basel (1981).

[31] E. T. Copson: On an integral equation arising in the theory of diffraction. Q. J. Math., Oxf. Ser. 17 (1946), $19-34$.

[32] V. G. Daniele: On the solution of two coupled Wiener-Hopf equations. SIAM J. Appl. Math. 44 (1984), 667-680.

[33] A. Devinatz, M. Shinbrot: General Wiener-Hopf operators. Trans. AMS 145 (1969), 467-494.

[34] R. Duduchava : Integral Equations with Fixed Singularities. Teubner, Leipzig (1979).

[35] R. Duduchava, F. -O. Speck: Bessel potential operators for the quarter-plane. Appl. Anal. 45 (1992), $49-68$.

[36] R. Duduchava, F. -O. Speck: Pseudodifferential operators on compact manifolds with Lipschitz boundary. Math. Nachr. 160 (1993), 149-191.

[37] R. Duduchava, W. L. Wendland: The Wiener-Hopf method for systems of pseudodifferential equations with an application to crack problems. Integr. Equ. Oper. Theory 23 (1995), 294-335.

[38] T. Ehrhardt, A. P. Nolasco and F. -O. Speck: Boundary integral methods for wedge diffraction problems: the angle 2 $\mathrm{pi} / \mathrm{n}$, Dirichlet and Neumann conditions. Operators and Matrices 5 (2011), 1-40.

[39] T. Ehrhardt, A. P. Nolasco and F. -O. Speck: A Riemannn surface approach for diffraction from rational wedges. Operators and Matrices 8 (2014), 301-355.

[40] T. Ehrhardt, F. -O. Speck: Transformation techniques towards the factorization of non-rational $2 x 2$ matrix functions. Linear Algebra Appl. 353 (2002), 53-90.

[41] G. I. Eskin: Boundary Value Problems for Elliptic Pseudodifferential Equations. AMS, Providence (1981), Russian edition 1973.

[42] I. C. Gohberg, I. A. Fel'dman: Convolution equations and projection methods for their solution. Translations of Mathematical Monographs 41. American Mathematical Society, Providence, R. I., (1974).

[43] I. Z. Gohberg, M. G. Krein: Systems of integral equations on a half-line with kernel depending on the difference of arguments. AMS Trans. 14 (1960), 217-287, Russian edition 1958.

[44] I. Gohberg, N. Krupnik: One-Dimensional Linear Singular Integral Equations I, II. Birkhäuser, Basel (1992), German edition 1979, Russian edition 1973.

[45] L. S. Goldenstein, I. C. Gohberg: On a multidimensional integral equation on a half-space whose kernel is a function on the difference of the arguments, and on a discrete analogue of this equation. Sov. Math. Dokl. 1 (1960), 173-176.

[46] P. Grisvard: Elliptic Problems in Nonsmooth Domains. Pitman, London, (1985).

[47] J. Hadamard: Sur les problèmes aux dérivées partielles et leur signification physique. In: Princeton University Bulletin 13, No. 4, (1902), 49-52.

[48] A. Heins: Systems of Wiener-Hopf integral equations and their application to some boundary value problems in electromagnetic theory. Proc. Symp. Appl. Math. 2 (1950), 76-81.

[49] A. Heins: The Sommerfeld half-plane problem revisited. I: The solution of a pair of coupled Wiener-Hopf integral equations. Math. Methods Appl. Sci. 4 (1982), 74-90.

[50] S. ter Horst, M. Messerschmidt and A. C. M. Ran: Equivalence after extension for compact operators on Banach spaces. J. Math. Anal. Appl. 431 (2015), 136-149.

[51] S. ter Horst, A. C. M. Ran: Equivalence after extension and matricial coupling coincide with Schur coupling, on separable Hilbert spaces. Linear Algebra Appl. 439 (2013), 793-805.

[52] G. C. Hsiao, W. L. Wendland: Boundary Integral Equations. Springer, Berlin (2008).

[53] R. A. Hurd: The Wiener-Hopf-Hilbert method for diffraction problems. Can. J. Phys. 54 (1976), 775-780.

[54] R. A. Hurd: The explicit factorization of $2 \times 2$ Wiener-Hopf matrices. Preprint 1040, Fachbereich Mathematik, Technische Hochschule Darmstadt (1987), 24 p.

[55] R. A. Hurd, E. Meister and F. -O. Speck :Sommerfeld diffraction problems with third kind boundary conditions. SIAM J. Math. Anal. 20 (1989), 589-607.

[56] M. Idemen: A new method to obtain exact solutions of vector Wiener-Hopf equations. Z. Angew. Math. Mech. 59 (1979), 656-658.

[57] D. S. Jones: A simplifying technique in the solution of a class of diffraction problems. Quart.J. Math. 3 (1952), $189-196$.

[58] D. S. Jones: The Theory of Electromagnetism. Pergamon Press, Oxford (1964).

[59] D. S. Jones: Commutative Wiener-Hopf factorization of a matrix. Proc. Roy. Soc. London, (1984), $185-192$. 
[60] A. A. Khrapkov: Certain cases of the elastic equilibrium of an infinite wedge with a nonsymmetric notch at the vertex subjected to concentrated forces. J. Appl. Math. Mech. 35 (1971), 625-637, English, translation from Prikl. Mat. Mekh. 35 (1971), 677-689, Russian.

[61] A. V. Kisil: Stability analysis of matrix Wiener-Hopf factorization of Daniele-Khrapkov class and reliable approximate factorization. Proc. A, R. Soc. Lond. 471 (2015), 15 p.

[62] M. G. Krein: Integral equations on a half-line with kernel depending on the difference of arguments. AMS Trans. 22 (1962), 163-288, Russian edition 1958.

[63] J. B. Lawrie, I. D. Abrahams: A brief historical perspective of the Wiener-Hopf technique. J. Eng. Math. 59 (2007), 351358.

[64] A. B. Lebre, A. Moura Santos and F. -O. Speck: Factorization of o class of matrices generated by Sommerfeld diffraction problems with oblique derivatives. Math. Meth. Appl. Sciences 20 (1997), 1185-1198.

[65] G. S. Litvinchuk, I. M. Spitkovskii: Factorization of Measurable Matrix Functions. Oper. Mathematical Research 37, Akademie-Verlag, Berlin (1987), and Theory Adv. Appl. 25, Birkhäuser, Basel (1987).

[66] E. Lüneburg: The Sommerfeld problem: Methods, generalization and frustrations. In: E. Meister (ed.), Modern Mathematical Methods in Diffraction Theory and its Applications in Engineering. Proceedings of the Sommerfeld '96 workshop. Peter Lang, Europ. Verlag der Wissenschaften, Frankfurt am Main (1997). Methoden Verfahren Math. Phys. 42, 145-162.

[67] E. Lüneburg, R. A. Hurd On the diffraction problem on a half-plane with different face impedances. Can. J. Phys. 62 (1984), 853-860.

[68] V. Maz'ya, T. Shaposhnikova: Jacques Hadamard, a universal mathematician. History of Mathematics 14, American Mathematical Society, Providence, RI (1998).

[69] E. Meister: Randwertaufgaben der Funktionentheorie. Mit Anwendungen auf singuläre Integralgleichungen und Schwingungsprobleme der mathematischen Physik. Leitfäden der Angewandten Mathematik und Mechanik 59. Teubner, Stuttgart (1983), German.

[70] E. Meister: Integraltransformationen mit Anwendungen auf Probleme der mathematischen Physik. Lang, Frankfurt (1983), German.

[71] E. Meister: Some solved and unsolved canonical problems of diffraction theory. In: Differential equations and mathematical physics, Proc. Int. Conf., Birmingham/Ala. (1986), Lect. Notes Math. 1285 (1987), 320-336. Short English, version of [72].

[72] E. Meister: Einige gelöste und ungelöste kanonische Probleme der mathematischen Beugungstheorie. Expo. Math. 5 (1987), 193-237, German.

[73] E. Meister (ed.): Modern Mathematical Methods in Diffraction Theory and its Applications in Engineering. Proceedings of the Sommerfeld' 96 workshop at Freudenstadt, Germany. Methoden und Verfahren der Mathematischen Physik 42. Peter Lang, Europ. Verlag der Wissenschaften, Frankfurt (1997).

[74] E. Meister, F. Penzel: On the reduction of the factorization of matrix functions of Daniele- Khrapkov type to a scalar boundary value problem on a Riemann surface. Complex Variables, Theory Appl. 18 (1992), 63-71.

[75] E. Meister, F. -O. Speck: Some multidimensional Wiener-Hopf equations with applications. In: Trends in applications of pure mathematics to mechanics, Vol. 2 (1979), Proceedings of a Symposium at Kozubnik Poland (1977), 217-262.

[76] E. Meister, F. -O. Speck: Diffraction problems with impedance conditions. Appl. Anal. 22 (1986), 193-211.

[77] E. Meister, F. -O. Speck: Modern Wiener-Hopf methods in diffraction theory. In: Ordinary and Partial Differential Equations, Vol. 2 Eds: B.D. Sleeman et al., Longman, London (1989), 130-171.

[78] E. Meister, F. -O. Speck: The explicit solution of elastodynamical diffraction problems by symbol factorization. Z. Anal. Anw. 8 (1989), 307-328.

[79] S. G. Mikhlin, S. Prössdorf: Singular Integral Operators. Springer, Berlin (1986), German edition: Akademie-Verlag, Berlin 1980.

[80] G. Mishuris, S. Rogosin: Factorization of a class of matrix-functions with stable partial indices. Math. Methods Appl. Sci. 39 (2016), 3791-3807.

[81] A. Moura Santos, F. -O. Speck: Sommerfeld diffraction problems with oblique derivatives. Math. Meth. Appl. Sciences 20 (1997), 635-652.

[82] A. Moura Santos, F. -O. Speck and F. S. Teixeira: Compatibility conditions in some diffraction problems. Pitman Research Notes in Mathematics Series 361, Longman, London (1996), 25-38.

[83] A. Moura Santos, F. -O. Speck and F. S. Teixeira: Minimal normalization of Wiener-Hopf operators in spaces of Bessel potentials. J. Math. Anal. Appl. 225 (1998), 501-531.

[84] R. J. Nagem, M. Zampolli and G. Sandri: Mathematical Theory of Diffraction. Birkhäuser, Boston (2004).

[85] M. Z. Nashed, L. B. Rall: Annotated bibliography on generalized inverses and applications. In: Generalized Inverses and Applications (Ed: M.Z. Nashed), Academic Press, New York (1976), 771-1041.

[86] B. Noble: Methods Based on the Wiener-Hopf Technique. Pergamon Press, London (1958). 
[87] F. Penzel, F. -O. Speck: Asymptotic expansion of singular operators on Sobolev spaces. Asymptotic Anal. 7 (1993), 287-300.

[88] H. Poincaré: Sur la polarisation par diffraction. Acta Math. 16 (1892), 297-339, French.

[89] L. Primachuk, S. Rogosin Factorization of triangular matrix-functions of an arbitrary order. Lobachevskii Journal of Mathematics 39 (2018), 809-817.

[90] S. Prössdorf, F. -O. Speck A factorisation procedure for two by two matrix functions on the circle with two rationally independent entries. Proc. R. Soc. Edinb., Sect. A 115 (1990), 119-138.

[91] A. D. Rawlins: The solution of a mixed boundary value problem in the theory of diffraction by a semi-infinite plane. Proc. Roy. Soc. London A 346 (1975), 469-484.

[92] A. D. Rawlins: The explicit Wiener-Hopf factorisation of a special matrix. Z. Angew. Math. Mech. 61 (1981), $527-528$.

[93] A. D. Rawlins: The solution of a mixed boundary value problem in the theory of diffraction. J. Eng. Math. 18 (1984), $37-62$.

[94] A. D. Rawlins, W. E. Williams: Matrix Wiener-Hopf factorisation. Q. J. Mech. Appl. Math. 34 (1981), 1-8.

[95] S. Rogosin, G. Mishuris Constructive methods for factorization of matrix-functions. IMA J. Appl. Math. 81 (2016), 365-391.

[96] A. F. dos Santos, F. S. Teixeira: The Sommerfeld problem revisited: Solution spaces and the edge conditions. J. Math. Anal. Appl. 143 (1989), 341-357.

[97] T. B. A. Senior: Diffraction by a semi-infinite metallic sheet. Proc. R. Soc. Lond., Ser. A 213 (1952), $436-458$.

[98] A. H. Serbest (ed.), S. R. Cloude (ed.): Direct and inverse electromagnetic scattering. Proceedings of the workshop, September 24-30, (1995), Gebze, Turkey. Mathematics Series 361. Longman, Harlow (1996).

[99] E. Shamir: Mixed boundary value problems for elliptic equations in the plane. The $L^{p}$ theory. Ann. Sc. Norm. Super. Pisa, Sci. Fis. Mat., III. Ser. 17 (1963), 117-139.

[100] E. Shamir Elliptic systems of singular integral operators. I. The half-space case. Trans. AMS 127 (1967), 107-124.

[101] M. Shinbrot: On singular integral operators. J. Math. Mech. 13 (1964), 395-406.

[102] ] I. B. Simonenko: Some general questions in the theory of the Riemann boundary problem. Izv. Akad. Nauk SSSR, Ser. Mat. 32 (1968), 1138-1146, Russian. Math. USSR, Izv. 2 (1970), 1091-1099, English.

[103] A. Sommerfeld: Mathematische Theorie der Diffraction. Math. Ann. 47 (1896), 317-374, German. Annotated translation in English see [84].

[104] A. Sommerfeld: Partial differential equations in physics. Academic Press, New York (1949).

[105] F. -O. Speck: On the generalized invertibility of Wiener-Hopf operators in Banach spaces. Integr. Equ. Oper. Theory 6 (1983), 458-465.

[106] F. -O. Speck: General Wiener-Hopf Factorization Methods. Pitman, London (1985).

[107] F. -O. Speck: Mixed boundary value problems of the type of Sommerfeld half-plane problem. Proc. Royal Soc. Edinburgh 104 A (1986), 261-277.

[108] F. -O. Speck: Sommerfeld diffraction problems with first and second kind boundary conditions. SIAM J. Math. Anal. 20 (1989), 396-407.

[109] F. -O. Speck: In memory of Erhard Meister. In: Operator Theoretical Methods and Applications to Mathematical Physics I, Eds. I. Gohberg et al., Oper. Theory Adv. Appl. 147, Birkhäuser, Basel (2004), 27-46.

[110] F. -O. Speck: On the reduction of linear systems related to boundary value problems. In: Operator theory, pseudodifferential equations, and mathematical physics, Eds. Yu.I. Karlovich et al., The Vladimir Rabinovich anniversary volume. Oper. Theory Adv. Appl. 228, Birkhäuser, Basel (2013), 391-406.

[111] F. -O. Speck: Diffraction from a three-quarter-plane using an abstract Babinet principle. Z. Angew. Math. Mech. 93 (2013), 485-491.

[112] F. -O. Speck: Wiener-Hopf factorization through an intermediate space. Integr. Equ. Oper. Theory 82 (2015), $395-415$.

[113] F. -O. Speck: A class of interface problems for the Helmholtz equation in $\mathbb{R}^{n}$. Math. Meth. Appl. Sciences 40 (2017), 391-403.

[114] F. -O. Speck: Paired operators in asymmetric space setting. In: Large Truncated Toeplitz Matrices, Toeplitz Operators, and Related Topics, Eds. D. Bini et al., The Albrecht Böttcher Anniversary Volume. Oper. Theory Adv. Appl. 259, Birkhäuser, Basel (2017), 681-702.

[115] F. -O. Speck: On the reduction of general Wiener-Hopf operators. In: Operator Theory, Analysis, and the State Space Approach, Eds. H. Bart et al., In Honor of Rien Kaashoek. Oper. Theory Adv. Appl. 271, Birkhäuser, Basel (2018), 399-419.

[116] I. M. Spitkovsky, A. M. Tashbaev: On the problem of effective factorization of matrix functions. Izv. Vyssh. Uchebn. Zaved., Mat. 4 (1989), 69-76, Russian. Sov. Math. 33 (1989), 85-93, English.

[117] G. Talenti: Sulle equazioni integrali di Wiener-Hopf. Boll. Unione Mat. Ital., IV. Ser. 7, Suppl. al Fasc. 1 (1973), 18-118, Italian.

[118] D. Timotin: Schur coupling and related equivalence relations for operators on a Hilbert space. Linear Algebra Appl. 452 (2014), 106-119.

[119] H. Triebel: Theory of function spaces. Akademische Verlagsgesellschaft, Leipzig (1983). 
[120] M. I. Vishik, G. I. Eskin: Equations in convolutions in a bounded region. Russian Math. Surveys 20 (1965), 85-151. Translation from Usp. Mat. Nauk 20 (1965), 89-152.

[121] L. A. Weinstein: The Theory of Diffraction and the Factorization Method. Golem Press, Boulder, Colorado (1969).

[122] N. Wiener, E. Hopf: Über eine Klasse singulärer Integralgleichungen. Sitzungsber. Preuß. Akad. Wiss., Phys.-Math. K1. 30-32 (1931), 696-706, German.

[123] W. E. Williams: Recognition of some readily "Wiener-Hopf" factorizable matrices. IMA J. Appl. Math. 32 (1984), 367-378.

[124] J. Wloka: Partial Differential Equations. Cambridge University Press, (1987).

DEPARTAMENTO DE MATEMÁticA

INSTITUTO SUPERIOR TÉCNICO

AVEnida Rovisco PAis, P 1049-001, Lisboa,Portugal

ORCID: 0000-0002-4246-3439

Email address: fspecketecnico.pt 